\title{
INTERNAL STELLAR KINEMATICS OF M32 FROM THE SPLASH SURVEY: DARK HALO CONSTRAINTS*
}

\author{
K. M. Howley ${ }^{1}$, P. Guhathakurta ${ }^{2}$, R. van der Marel ${ }^{3}$, M. Geha ${ }^{4}$, J. Kalirai $^{3}$, B. Yniguez ${ }^{5}$, \\ E. Kirby ${ }^{6,9}$, J.-C. CuillandRE ${ }^{7}$, AND K. GILBERT ${ }^{8,9}$ \\ ${ }^{1}$ Lawrence Livermore National Laboratory, P.O. Box 808, Livermore, CA 94551, USA; howley1@1lnl.gov \\ ${ }^{2}$ UCO/Lick Observatory, University of California Santa Cruz, 1156 High Street, Santa Cruz, CA 95064, USA; raja@ ucolick.org \\ ${ }^{3}$ Space Telescope Science Institute, 3700 San Martin Drive, Baltimore, MD 21218, USA; marel@ stsci.edu, jkalirai@ stsci.edu \\ ${ }^{4}$ Department of Astronomy, Yale University, New Haven, CT 06510, USA; marla.geha@yale.edu \\ ${ }^{5}$ Physics \& Astronomy Department, University of California, Irvine, 4129 Frederick Reines Hall, Irvine, CA 92697, USA; byniguez@uci.edu \\ ${ }^{6}$ Department of Astronomy, California Institute of Technology, 1200 East California Blvd, Pasadena, CA 91125, USA; enk@ astro.caltech.edu \\ ${ }^{7}$ Canada-France-Hawaii Telescope, 65-1238 Mamalahoa Hwy, Kamuela, HI 96743, USA; jcc@ cfht.hawaii.edu \\ ${ }^{8}$ Department of Astronomy, University of Washington, Box 351580, Seattle, WA 98195, USA; kgilbert@ astro.washington.edu \\ Received 2012 February 10; accepted 2012 November 19; published 2013 February 15
}

\begin{abstract}
As part of the SPLASH survey of the Andromeda (M31) system, we have obtained Keck/DEIMOS spectra of the compact elliptical (cE) satellite M32. This is the first resolved-star kinematical study of any cE galaxy. In contrast to most previous kinematical studies that extended out to $r \lesssim 30^{\prime \prime} \sim 1 r_{I}^{\text {eff }} \sim 100 \mathrm{pc}$, we measure the rotation curve and velocity dispersion profile out to $r \sim 250^{\prime \prime}$ and higher order Gauss-Hermite moments out to $r \sim 70^{\prime \prime}$. We achieve this by combining integrated-light spectroscopy at small radii (where crowding/blending are severe) with resolved stellar spectroscopy at larger radii, using spatial and kinematical information to account statistically for M31 contamination. The rotation curve and velocity dispersion profile extend well beyond the radius $\left(r \sim 150^{\prime \prime}\right)$ where the isophotes are distorted. Unlike NGC 205, another close dwarf companion of M31, M32's kinematics appear regular and symmetric and do not show obvious sharp gradients across the region of isophotal elongation and twists. We interpret M31's kinematics using three-integral axisymmetric dynamical equilibrium models constructed using Schwarzschild's orbit superposition technique. Models with a constant mass-to-light ratio can fit the data remarkably well. However, since such a model requires an increasing tangential anisotropy with radius, invoking the presence of an extended dark halo may be more plausible. Such an extended dark halo is definitely required to bind a half-dozen fast-moving stars observed at the largest radii, but these stars may not be an equilibrium component of M32.
\end{abstract}

Key words: galaxies: dwarf - galaxies: individual (M32, NGC 221) - galaxies: kinematics and dynamics Local Group - techniques: radial velocities - techniques: spectroscopic

Online-only material: color figures

\section{INTRODUCTION}

Andromeda's (M31) nearest companion, M32, is our closest example of a compact elliptical (cE), a rare dwarf galaxy type. Including M32, there are fewer than ten $\mathrm{cE}$ galaxies known within 100 Mpc (Rood 1965; King \& Kiser 1973; Smith Castelli et al. 2009; Chilingarian et al. 2009; Chilingarian \& Bergond 2010). Compact elliptical galaxies have luminosities of $\sim 10^{9} L_{\odot}$, comparable to dwarf elliptical galaxies $(\mathrm{dE})$, but with significantly smaller effective radii $\left(r_{\text {eff }} \sim\right.$ $0.1-0.7 \mathrm{kpc}$ ), leading to notably higher surface brightnesses ( $\mu_{\text {eff }} \sim 18-21 \mathrm{mag} \operatorname{arcsec}^{-2}$, Choi et al. 2002; Mieske et al. 2005; Chilingarian et al. 2009). cEs appear to be a rare galaxy type, and the discovery of these objects has been slow due to their elliptical galaxy-like appearance at distances beyond the Local Group (Ziegler \& Bender 1998; Drinkwater \& Gregg 1998).

Compact elliptical galaxies are consistently found in close projection to a massive parent galaxy $\left(5<r_{\text {proj }}<80 \mathrm{kpc}\right.$, Chilingarian et al. 2009), indicating that gravitational effects play some role in their evolution. One formation scenario

\footnotetext{
* Data herein were obtained at the W. M. Keck Observatory, which is operated as a scientific partnership among the California Institute of Technology, the University of California, and NASA. The Observatory was made possible by the generous financial support of the W. M. Keck Foundation.

9 Hubble Fellow.
}

proposes that cEs are remnant cores of tidally stripped "normal" galaxies (Faber 1973; Nieto 1990; Bekki et al. 2001; Graham 2002; Chilingarian \& Bergond 2010; Huxor et al. 2010), although the rarity of cEs relative to normal elliptical and spiral galaxies suggests that this does not happen often, or that cEs are short-lived. Another formation theory suggests that cEs are lowmass classical elliptical galaxies that were either captured by or formed in the potential well of a massive neighbor (Nieto \& Prugniel 1987; Burkert 1994). This latter scenario is supported by the position of cEs on the fundamental plane, at the low luminosity end of the classical elliptical galaxy trend (Wirth \& Gallagher 1984; Kormendy 1985; Nieto \& Prugniel 1987). If these objects are indeed low mass normal elliptical galaxies, then their rarity implies a steep fall off at the faint end of the galaxy luminosity function (Binggeli et al. 1988). Recent observational improvements indicate that the light profiles of many cEs, including M32, are better fit by a two component $r^{1 / n}$ bulge + exponential profile, characteristic of a disk galaxy, rather than the historically used $r^{1 / 4}$ law, typical of elliptical galaxies (Graham 2002).

As the nearest example of the cE class, M32 is an excellent specimen for examining $\mathrm{cE}$ properties. As is the case for other cEs, M32 lies close to its parent galaxy at a projected separation of only $22^{\prime}(5 \mathrm{kpc}$ ) from M31's center. Our relative proximity to M32 allows us to resolve individual stars in this object—at least for outer radii where stellar crowding is less severe. Photometric 
evidence suggests that M32's physical distance from M31 is similar to its projected distance: Choi et al. (2002) show that distortions in M32's outer elliptical isophotes are consistent with the hypothesis that these two galaxies are tidally interacting. Tidal interactions are a possible explanation for the galaxy's unusual stellar population gradient and light profile (Faber 1973; O'Connell 1980; Davidge 2000; Davidge et al. 2000; Bekki et al. 2001; Graham 2002). Integrated light spectroscopic studies suggest the presence of a younger, more metal-rich stellar population at the center of M32 (Rose et al. 2005; Coelho et al. 2009 and references therein) which is perhaps the result of a tidally induced nuclear starburst (Bekki et al. 2001).

Previous studies of M32's internal kinematics have primarily focused on the inner regions. The steep rotational velocity gradient at the center of M32 indicates the presence of a central dark mass. High resolution imaging and integrated light spectroscopy have confirmed the presence of a central black hole with mass $M_{\mathrm{BH}} \sim 2-4 \times 10^{6} M_{\odot}$ (Goodman \& Lee 1989; Bender et al. 1996; van der Marel et al. 1997, 1998a; Joseph et al. 2001; Verolme et al. 2002; Tremaine et al. 2002; van den Bosch $\&$ de Zeeuw 2010). While these studies have been appropriate for determining the mass of the central dark object, their limited radial extent (generally $r<10^{\prime \prime}$ ) does not provide much information on the wider galaxy environment. Past attempts to study the kinematics of M32 at larger radii $\left(r<60^{\prime \prime}\right)$ have produced conflicting results (Tonry 1984; Carter \& Jenkins 1993; Toloba et al. 2011). Specifically, Tonry (1984) and Toloba et al. (2011) found the velocity dispersion to increase outward while Carter \& Jenkins (1993) found it to be decrease outward. The authors profess problems in their measurements due to complicated sky subtraction and, in particular, contamination from M31.

In this paper, we use a combination of integrated light and resolved stellar spectroscopy to obtain an accurate kinematical profile of M32 out to $\sim 8 r_{\text {eff }}$ - a much larger radius than has been previously possible. In the inner regions of M32, crowding is so significant that individual stars cannot be resolved, while in the outer regions, the integrated light spectrum becomes very noisy due to the presence of M31 and the steep light profile of M32. This is the first attempt to combine spectra of individual stars with integrated light spectroscopy to obtain a complete picture of M32. This work is part of the SPLASH Survey (Spectroscopic and Photometric Landscape of Andromeda's Stellar Halo), aimed at the study of M31 and its satellites.

This paper is organized as follows. In Section 2, we measure the internal kinematics of M32 out to a radius of $\sim 8 r_{\text {eff }}$ $(\sim 1 \mathrm{kpc})$ using resolved stellar light and $\sim 3 r_{\text {eff }}(\sim 0.3 \mathrm{kpc})$ using integrated light. We then present velocity, velocity dispersion, and Gauss-Hermite moment major- and minor-axis profiles from these measurements and compare them to previous measurements. Using these results, we construct an axisymmetric, three-integral model in Section 3 to obtain an estimate of M32's mass and mass-to-light ratio $(M / L)$. Finally, in Section 4, we summarize and discuss our results.

Throughout this paper, we assume that M32 has the same distance modulus as M31: $(m-M)_{0}=20.54 \pm 0.03(785 \pm$ $25 \mathrm{kpc}$, McConnachie et al. 2005). At M32/M31's distance, $1^{\prime \prime}$ is equivalent to $3.8 \mathrm{pc}$. While the distance to M32 has been measured independently (Jensen et al. 2003; Karachentsev et al. 2004), it is consistent with the distance to M31. Moreover, the M32 distance estimate is affected by crowding problems and M31 contamination. The lack of obvious signs of dust extinction in M32 suggests that the satellite lies in front of M31's disk
(Ford et al. 1978), but a precise M31-M32 distance is yet to be established.

\section{STELLAR KINEMATICS OF M32}

In this section, we give a detailed account of our M32 kinematical measurements. In Section 2.1, we present measurements from the resolved stellar kinematics. In Section 2.2, we present measurements from integrated light. For readers not interested in these details, an integrated view of M32's kinematics and a comparison to them are presented in Section 2.3.

\subsection{Resolved Stellar Population Spectroscopy}

In this subsection, we discuss our observational setup, including photometric and astrometric measurements (Section 2.1.1), mask placement (Section 2.1.2), identification of isolated sources (Section 2.1.3), target selection and slit mask design (Section 2.1.4), and observing details (Section 2.1.5). We then discuss the reduction and analysis of the spectra, including the data reduction process (Section 2.1.6), measurement of individual stellar velocities (Section 2.1.7), quality assessment (Section 2.1.8), and detection of and velocity measurements for serendipitously detected stars (Section 2.1.9). Finally, in Section 2.1.10, we make velocity and velocity dispersion measurements along M32's major and minor axes via maximum likelihood analysis.

\subsubsection{Pre-imaging}

We derive photometric and astrometric catalogs of stars in M32's general vicinity from archival data obtained with the $\mathrm{MegaCam}^{10}$ imager on the $3.6 \mathrm{~m}$ Canada-France-Hawaii Telescope (CFHT). These data are in the form of $2^{\circ} \times 2^{\circ}$ mosaic images in the $g^{\prime}, r^{\prime}$, and $i^{\prime}$ bands centered on M31.

The mosaic image in each band was built from four separate MegaCam pointings, each with a field of view of $1^{\circ} \times 1^{\circ}$. Each MegaCam pointing consists of five dithered, slightly overlapping exposures with integration times of $5 \times 45 \mathrm{~s}$ in each of the $g^{\prime}$ and $r^{\prime}$ bands and $5 \times 90 \mathrm{~s}$ in the $i^{\prime}$ band. Thus, the effective integration times are $225 \mathrm{~s}$ in each of the $g^{\prime}$ and $r^{\prime}$ bands and $450 \mathrm{~s}$ in the $i^{\prime}$ band.

The data were obtained under photometric conditions. Individual exposures were detrended using CFHT's data reduction pipeline, Elixir, and stacked into mosaic images using the SWarp software (Bertin et al. 2002). The pixel scale on the mosaic image is 0.36 , a factor of 2 coarser than the native MegaCam pixel scale. The median seeing FWHM is $1^{\prime \prime} .2,1^{\prime \prime} .0$ and $0^{\prime \prime} .7$ in $g^{\prime}, r^{\prime}$, and $i^{\prime}$, respectively. In order to highlight point-like sources, a highpass filtered image is constructed by subtracting a smoothed version of the mosaic image from the original.

For the purposes of this M32 study, we analyze a $36^{\prime} \times 36^{\prime}$ section of the $i^{\prime}$ and $r^{\prime}$ mosaic images centered on M32 (given the high degree of crowding in this region, the $g^{\prime}$ image is not particularly useful due to its relatively poor seeing and the $r^{\prime}$ image is only used in the construction of Figure 3; neither the $g^{\prime}$ nor $r^{\prime}$ images are used for spectroscopic selection). The size of this image section is chosen to comfortably allow for the

\footnotetext{
10 http://www.cfht.hawaii.edu/Instruments/Imaging/MegaPrime/; MegaPrime/MegaCam is a joint project of CFHT and CEA/DAPNIA at the CFHT which is operated by the National Research Council (NRC) of Canada, the Institut National des Science de l'Univers of the Centre National de la Recherche Scientifique (CNRS) of France, and the University of Hawaii. The observations were obtained by the MegaCam instrument team in 2004 November.
} 


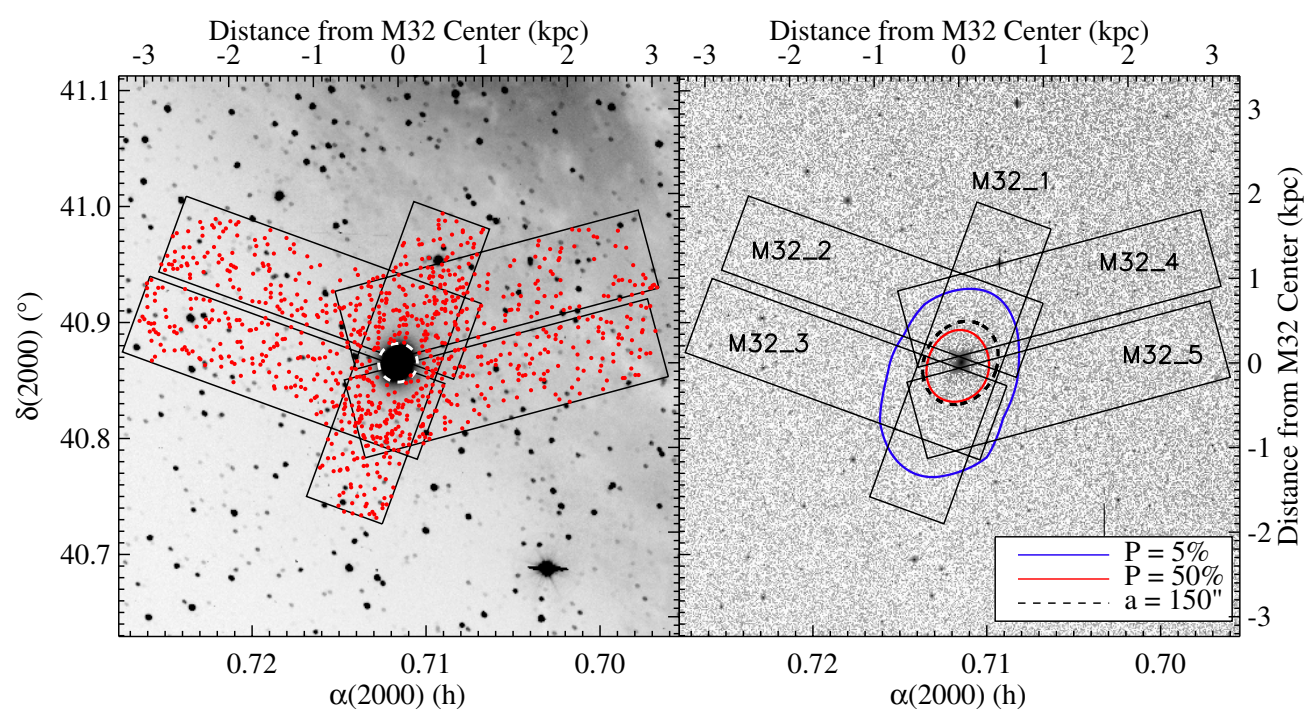

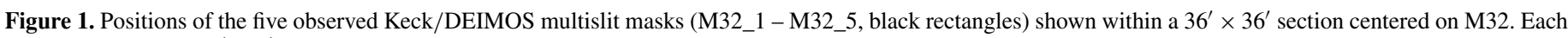

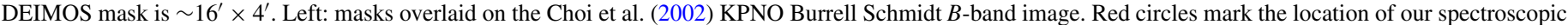

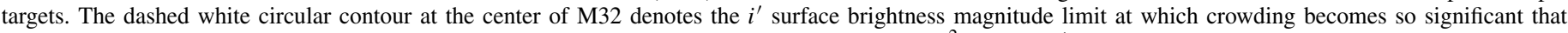

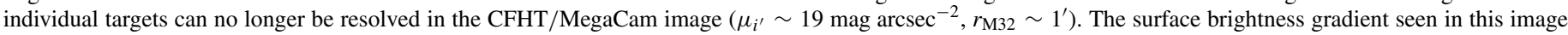

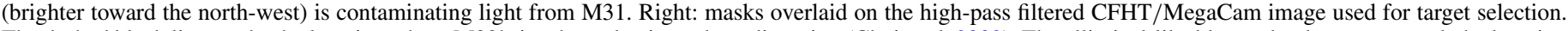

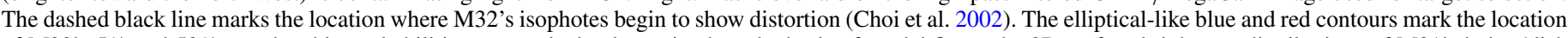

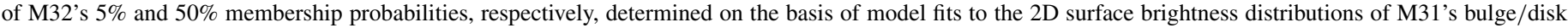
and M32.

(A color version of this figure is available in the online journal.)

placement of multiple Keck/DEIMOS multislit spectroscopic masks (each mask covers about $16^{\prime} \times 4^{\prime}$ ), as shown in Figure 1 .

We generate star lists over the high-pass filtered $r^{\prime}$ and $i^{\prime}$ MegaCam mosaic images using the FIND task in the DAOPHOT photometry package (Stetson 1994) and carry out preliminary aperture photometry of all detected sources. A spatially varying point-spread function (PSF) template is iteratively derived from a set of bright, isolated stars whose neighbors have been subtracted using these star lists. This PSF template is then fit to all sources in the catalog for each frame using the ALLSTAR module to produce accurate photometric catalogs. Best-fit PSF templates are then subtracted from the high-pass filtered image to create a subtracted image that shows the residuals due to imperfect PSF subtraction and missed sources. The source find procedure and photometry procedures are then repeated on the PSF subtracted images several times to identify faint objects missed in earlier passes, and this yields a final photometric catalog of over $10^{5}$ stars. Next, the magnitudes are roughly calibrated based on the tip of the red giant branch (TRGB) in each filter; precise photometric calibration is not needed for this project since the purpose of our photometric catalog is solely to assign rough $i^{\prime}$ magnitude ranges from which to select and set priorities for spectroscopic targets. As a final step, we astrometrically correct all of our $x$ and $y$ positions by computing transformations based on the USNO A2.0 catalog. The final RA and DEC of all of the sources are accurate to $\sim 0^{\prime} \cdot 2$. We demonstrate our photometric characterization of sources in the $i^{\prime}$ image by illustrating both the original and PSF subtracted images in the vicinity of M32 in Figure 2.

Figure 3 shows the $i^{\prime}$ and $r^{\prime}$ photometry for stars within the 5\% membership probability contour centered on M32 (see Figure 1, right). Note that the color-magnitude diagrams (CMDs) in Figure 3 are limited by the poor quality of the $r^{\prime}$ data, and are therefore used here for illustration purposes only. The plots on the left show the photometry of targets in the velocity range of M32 $\left(-275<v<-125 \mathrm{~km} \mathrm{~s}^{-1}\right.$; see Section 2.1.10), while the figures on the right plot the remaining non-M32 like targets. All four CMDs look very similar, thus illustrating the difficulty of photometrically preselecting stars that are likely to be M32 members. At best, the CMDs can be used to preselect stars at or below the TRGB $\left(i^{\prime} \geqslant 20\right)$, and above our spectroscopic limit $\left(i^{\prime} \leqslant 22\right)$.

\subsubsection{Mask Placement}

The two largest obstacles to spectroscopy of the resolved stellar population of M32 are contamination by M31 and crowding. We consider each of these in turn.

The probability of a star being an M32 member can be estimated by the M32/M31 surface brightness ratio at that location. The two-dimensional surface brightness of M32 is modeled as a series of concentric ellipses based on the Choi et al. (2002) measurements of $I$-band surface brightness, ellipticity, and position angle as a function of radius. The two-dimensional surface brightness of M31 is modeled as the sum of a Sersíc bulge and an exponential disk based on published $V$-band surface brightness data and a mean color $V-I=1.6$ (Walterbos \& Kennicutt 1987; Pritchet \& van den Bergh 1994; Guhathakurta et al. 2005; Irwin et al. 2005). The 5\% and 50\% M32/(M31 + M32) I-band surface brightness ratios are shown in Figure 1 (for a more detailed mapping see Figure 5). The probability of M32 membership drops off very rapidly with increasing radius because of M32's steep surface brightness profile.

Near the center of M32, however, it is difficult, if not impossible, to find isolated spectroscopic targets in the highpass filtered CFHT image. Even the most luminous RGB stars (that are otherwise ideal for spectroscopy) are badly blended. Based on visual inspection (see Section 2.1.3), we avoid the inner $r_{\mathrm{M} 32} \lesssim 1^{\prime}$ region, corresponding to a surface brightness of $\mu_{I}=18.9 \mathrm{mag} \mathrm{arcsec}^{-2}$. 




Figure 2. Example of the CFHT/MegaCam image quality in various regions. Left: outline of the M32_1 DEIMOS mask (grey) on the high-pass filtered CFHT/MegaCam image with three 0.7 $\times 1$ 1.0 blue boxes defining representative areas zoomed into on the right. Middle: the column of plots demonstrates the quality of the image and the density of targets at the various locations on the mask. The selections show: (top) region near M32, and (bottom) the southern part of the mask. The red circles mark the location of the sources detected in the photometry. Right: the PSF subtracted images.

(A color version of this figure is available in the online journal.)

The arrangement of our five Keck/DEIMOS multislit masks is shown in Figure 1. Each mask covers $\approx 16^{\prime} \times 4^{\prime}$. The masks provide coverage of the $r_{\mathrm{M} 32}<150^{\prime \prime}$ region where M32's isophotes are regular, and the $r_{\mathrm{M} 32}>150^{\prime \prime}$ region where isophotal distortion is observed (Choi et al. 2002). The first slitmask, M32_1, is centered on M32 with the long axis rotated to a position angle $\phi$ of $160^{\circ}$, i.e., the approximate position angle of the inner elliptical isophotes (Choi et al. 2002). The remaining four multislit masks, M32_2-M32_5, are oriented to optimize coverage of the outer regions. M32's steep brightness profile implies that there is only a limited region in which neither contamination nor crowding is too severe; our arrangement of masks ensures good coverage of this region, while also covering M31's inner spheroid and disk for other SPLASH survey science.

\subsubsection{Identifying Isolated Sources}

Our chosen placement of the five Keck/DEIMOS masks defines the footprint over which spectroscopic targets are selected. We next identify the stars within this footprint that are least affected by crowding/blending. We use two criteria to reject targets: (1) cases where DAOPHOT finds one or more bright neighbors that are close to, but distinct from, the target (we refer to this as crowding), and (2) cases where an apparent single source in the DAOPHOT catalog is a poor fit to the PSF (we refer to this as blending).

We address the issue of crowding by rejecting a target star if it has a neighbor in the DAOPHOT catalog that is so close/bright that the PSF of the neighbor significantly overlaps that of the target. Based on visual inspection of the crowded regions of the image, we have come up with an empirical criterion. Any target that has even a single neighbor satisfying the following relation is eliminated from the list of potential spectroscopic targets:

$$
I_{\mathrm{tgt}}>I_{\mathrm{nbr}}+\frac{\left|\boldsymbol{r}_{\mathrm{tgt}, \mathrm{nbr}}\right|}{0.8}-3.0
$$

where $I_{\mathrm{tgt}}$ and $I_{\mathrm{nbr}}$ are the apparent magnitudes of the target and neighbor, respectively, and $\boldsymbol{r}_{\mathrm{tgt}, \mathrm{nbr}}$ is the projected distance between the two objects. Of the sources in the DAOPHOT catalog in the magnitude range $I=20-22$ (the range used to select spectroscopic targets, see Section 2.1.4), $\approx 10 \%$ pass this crowding test; the surviving fraction increases with target brightness over this magnitude range.

We address the issue of blending by visually inspecting the images at the locations of the stars that survive the crowding test. This inspection includes both the high-pass filtered and PSF-subtracted versions of the $i^{\prime}$-band CFHT/MegaCam image, as illustrated in Figure 2. Each target is flagged as unblended 


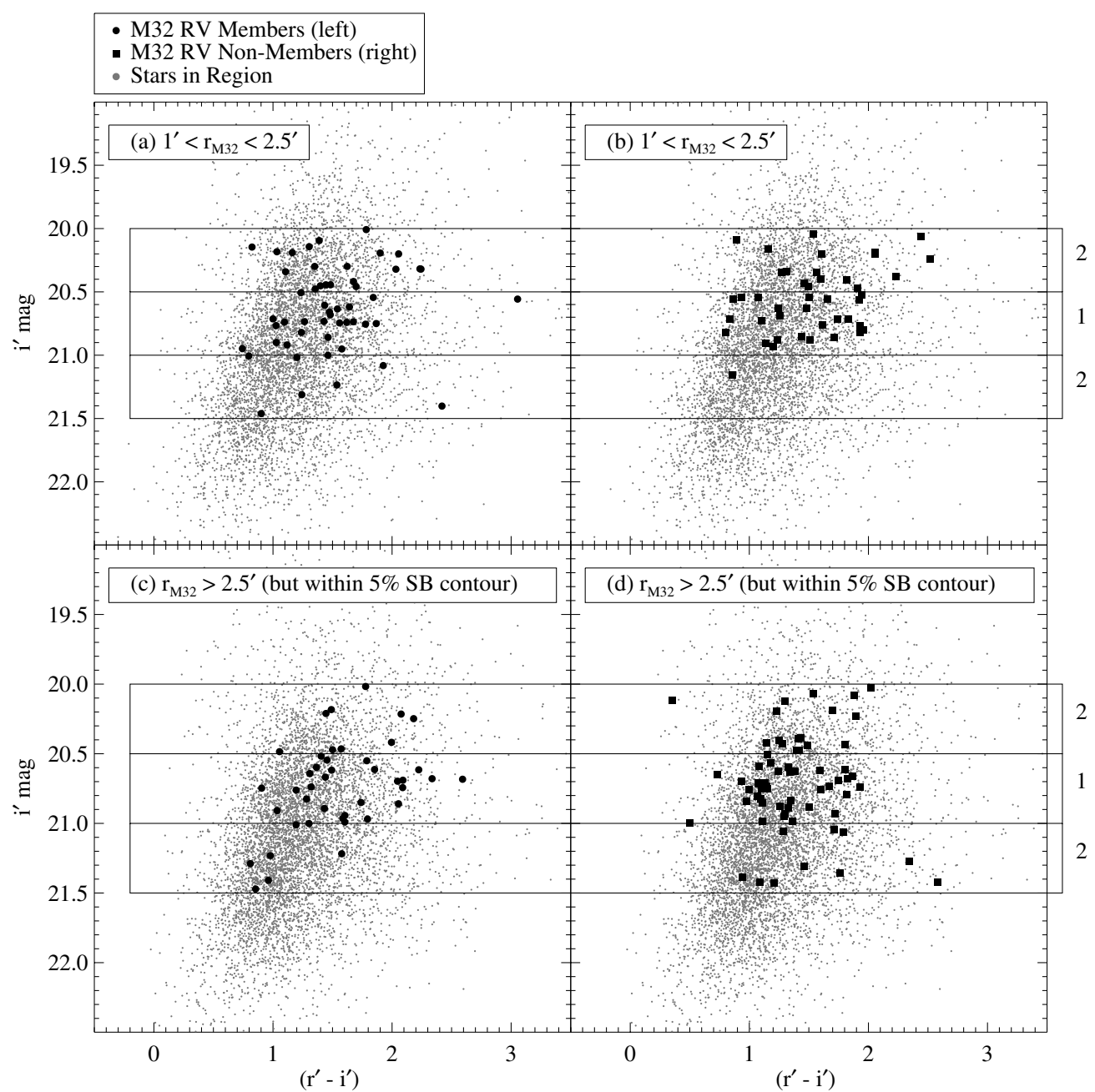

Figure 3. Color-magnitude diagram (CMD) based on the $i^{\prime}$ and $r^{\prime}$ photometry from the high-pass filtered CFHT MegaCam image (for illustration only due to the poor quality of the $r^{\prime}$ data). Stars shown are within the 5\% M32 membership probability contour (see Figure 1, right) and outside 1' of M32's severely crowded center (see Figure 1, left). The absolute calibration of the data is approximate and based on the TRGB magnitudes for M31 from McConnachie et al. (2005). The black circles/squares denote Keck/DEIMOS spectroscopic targets with measured velocities. The numbered rectangular outlines mark the location of our spectroscopic priorities: (1) primary and (2) secondary. Top Left: black circles show M32 candidate members (i.e., stars with velocities in the range $-275 \leqslant v \leqslant-125 \mathrm{~km} \mathrm{~s}^{-1}$ ) within $1^{\prime}<r_{\mathrm{M} 32}<2$ '.5. Top Right: black squares show M32 non-members (i.e., stars with velocities in the range $v<-275 \mathrm{~km} \mathrm{~s}^{-1}$ or $v>-125 \mathrm{~km} \mathrm{~s}^{-1}$ ) within $1^{\prime}<r_{\mathrm{M} 32}<2$ '.5. Bottom Left: black circles show M32 candidate members with $r_{\mathrm{M} 32}>2$ '.5 (but within M32's 5\% probability contour, i.e., $r_{\mathrm{M} 32}<5$ '.3). Bottom Right: black squares show M32 non-members with $r_{\mathrm{M} 32}>2$ '.5 (but within M32's 5\% probability contour, i.e., $r_{\mathrm{M} 32}<5.3$ ). Note the similarity between all four populations, thereby illustrating the difficulty of photometrically disentangling these two populations.

(high priority), marginally blended (medium priority), or badly blended (low priority), depending on the degree to which its image resembles the PSF on the high-pass filtered image and the strength of systematic residuals at its location on the PSF-subtracted image.

These de-blending exercises are only good to a point, as we are limited by the 0.'8 seeing (FWHM) of the CFHT/MegaCam image. The seeing FWHM was significantly better than this during the Keck/DEIMOS spectroscopic observations (Section 2.1.5). As a result, further de-blending of sources is carried out in the spatial and spectral domains, as discussed in Section 2.1.9. See Dorman et al. (2012) for a discussion of an automated procedure for identifying blended sources in this data set.

\subsubsection{Mask Design}

Five Keck/DEIMOS multislit masks are designed using A. C. Phillip's dsimulator software. ${ }^{11}$ Targets are prioritized

\footnotetext{
$\overline{11} \mathrm{http} / / /$ www.ucolick.org/ phillips/deimos_ref/masks.html
}

for spectroscopic observation based on two criteria: level of blending and magnitude (see Table 1). The software takes as input the multiple target lists (lists 1-3) organized by target priority (priorities $1-2$ ). Each $\approx 16^{\prime} \times 4^{\prime}$ Keck/DEIMOS mask is populated with targets from list 1 , in order of decreasing priority based on magnitude, followed by list 2 , and so on. Our selection process is identical to that discussed in Appendix A of Guhathakurta et al. (2006), with the following modifications: target prioritization, minimum distance between target and slit end (1".65), and distance between adjacent slitlets ( $\left.0{ }^{\prime \prime} 3\right)$. The location of the slitlets selected for observation is shown in Figure 1. The five masks contain a total of 883 slitlets.

\subsubsection{Observations}

The masks were observed between 2007 November and 2008 August using the DEIMOS spectrograph (Faber et al. 2003) on the Keck II $10 \mathrm{~m}$ telescope. The arrangement of the masks is shown in Figure 1. The observing details are summarized in Table 2. 
Table 1

Target Prioritization for Spectroscopic Observation

\begin{tabular}{ll}
\hline \hline First Level Prioritization & \multicolumn{1}{c}{ Level of Blending } \\
\hline List 1 (High Priority) & Unblended \\
List 2 (Medium Priority) & $\begin{array}{l}\text { Marginally blended } \\
\text { List 3 (Low Priority) }\end{array}$ \\
\hline Second Level Prioritization & I-band Magnitude Range \\
\hline Priority 1 & $20.5-21$ \\
Priority 2 & $20-20.5$ or 21-21.5 \\
Excluded & Targets outside 20-21.5 range \\
\hline
\end{tabular}

Notes. Target prioritization for spectroscopic observation based on the level of blending and magnitude. The first prioritization divides targets into three lists based on the level of blending (see Section 2.1.3). The second prioritization assigns priorities within each list based on M31's TRGB I-band magnitude of 20.5 (McConnachie et al. 2005), with the highest priority assigned to targets at or just below the TRGB. The distribution of targets across these magnitude ranges is shown in Figure 3.

All five multislit masks were observed with the 1200 line $\mathrm{mm}^{-1}$ grating. This configuration yields a spatial scale of 0 .'12 pixel $^{-1}$ and a spectral dispersion of $0.33 \AA \mathrm{pixel}^{-1}$. We set the central wavelength to $7800 \AA$, corresponding to a wavelength range of $\sim 6450-9150 \AA$. The exact wavelength range for each slit varies as a result of location on the multislit mask and/or truncation due to vignetting. The wavelength region is chosen to target several spectral features including the strong Ca II triplet absorption feature present in RGB stars. The anamorphic distortion factor for this grating and central wavelength is 0.606 . Therefore, the $0{ }^{\prime} .8$ wide slitlets subtend 4.1 pixels. Better still, excellent seeing conditions $\left(\sim 00^{\prime} 6\right)$ during observations provide somewhat better spectral resolution, yielding an average resolution of 3.1 pixels $=1.0 \AA$ FWHM.

Useful spectra are obtained from 786 of the 883 slitlets (89\%). The success rate would have been even higher were it not for the fact that $\approx 25 \%$ of the slitlets on mask M32_3 were lost due to buckling of the mask during its insertion into the DEIMOS focal plane at the time of observations; fortunately, the buckling took place at the ENE end of the mask away from M32.

\subsubsection{Data Reduction}

The masks were processed using the spec $2 d$ and specld software (version 1.1.4) developed by the DEEP Galaxy Redshift Survey team at the University of California, Berkeley. ${ }^{12}$ Briefly, the reduction pipeline rectifies, flat-field and fringe corrects,

12 http://astro.berkeley.edu/ cooper/deep/spec2d/ wavelength calibrates, sky subtracts, and cosmic ray cleans the two-dimensional spectra, and extracts the one-dimensional spectra. We give a more detailed description of the reduction process below.

First, the reduction pipeline rectifies curved spectra into rectangular arrays by applying small shifts and interpolating in the spatial direction. One-dimensional slit function, and two-dimensional flat-field and fringing corrections are then applied to the spectra. The wavelength solution of the rectified spectra is obtained by fitting a polynomial to the arc lamp lines (precise at the $0.01 \AA$ level). The two-dimensional spectra are then sky subtracted and cosmic ray cleaned. Sky is identified by collapsing the two-dimensional spectra in the wavelength direction to locate spatial positions along the slit that are least affected/unaffected by targets and serendipitous sources (see Section 2.1.9 for a discussion of serendipitous sources). Each two-dimensional spectrum is sky subtracted by fitting a B-spline model (wavelength as a function of two-dimensional position: $x, y)$ to the night sky emission lines in the baseline portion of the spatial intensity profile. This careful sky subtraction is of particular importance around the Ca II triplet region due to the presence of bright night sky lines; poor sky subtraction would reduce our ability to accurately measure stellar velocities. The two-dimensional exposures are then combined along with cosmic ray rejection and inverse variance weighting to create a single mean two-dimensional spectrum for each slit.

Last, the target is identified and its one-dimensional spectrum extracted. Targets are located on the two-dimensional spectrum by identifying the peak brightness distribution in spatial intensity profile, obtained by collapsing the two-dimensional spectrum in the wavelength direction. Target one-dimensional spectra are extracted from the two-dimensional spectra using a small spatial extraction window centered on the target. The onedimensional spectra are re-binned into logarithmic wavelength bins with $13.8 \mathrm{~km} \mathrm{~s}^{-1}$ pixel $^{-1}$. The final result is a wavelength calibrated, sky subtracted, cosmic ray cleaned one-dimensional spectrum for each target. An illustration of this process is shown in Figure 4(a).

\subsubsection{Cross Correlation Analysis}

Line-of-sight (LOS) velocities for resolved targets are measured from the one-dimensional spectra using a Simon \& Geha (2007) modified version of the visual inspection software zspec, developed by D. Madgwick for the DEEP Galaxy Redshift Survey at the University of California, Berkeley. The software determines the best-fit LOS velocity for a target by cross correlating its one-dimensional science spectrum with high signal-to-noise $(\mathrm{S} / \mathrm{N})$ stellar templates in pixel space and locating the best fit

Table 2

Keck/DEIMOS Multislit Mask Exposures

\begin{tabular}{|c|c|c|c|c|c|c|c|c|c|}
\hline $\begin{array}{l}\text { Mask } \\
\text { Name }\end{array}$ & $\begin{array}{l}\text { Observation } \\
\text { Date }\end{array}$ & $\begin{array}{c}\alpha \\
(\mathrm{J} 2000.0)\end{array}$ & $\begin{array}{c}\delta \\
(\mathrm{J} 2000.0)\end{array}$ & $\begin{array}{l}\text { P.A. } \\
\text { (deg) }\end{array}$ & $\begin{array}{l}t_{\exp } \\
(\mathrm{m})\end{array}$ & $\begin{array}{l}\text { Seeing } \\
\text { FWHM }\end{array}$ & $\begin{array}{l}\text { No. of } \\
\text { Slits }\end{array}$ & $\begin{array}{l}\text { No. of Useable } \\
\text { Target Velocities }\end{array}$ & $\begin{array}{c}\text { No. of Useable } \\
\text { Serendip Velocities }\end{array}$ \\
\hline M32_1 ${ }^{\mathrm{a}}$ & 2007 Nov 14 & 004238.3 & +405134.0 & 160 & $2 \times 20$ & 0.5 & 194 & $189(97 \%)$ & 73 \\
\hline M32_2 & 2008 Aug 3 & 004303.8 & +405507.7 & 70 & $3 \times 20$ & 0.6 & 184 & $169(92 \%)$ & 17 \\
\hline M32_3 & 2008 Aug 3 & 004311.6 & +405234.7 & -110 & $3 \times 20$ & 0.7 & 191 & $134(70 \%)^{b}$ & 10 \\
\hline M32_4 & 2008 Aug 4 & 004213.9 & +405444.2 & 105 & $3 \times 20$ & $0 . .6$ & 143 & $137(96 \%)$ & 117 \\
\hline M32_5 & 2008 Aug 4 & 004213.9 & +405202.6 & -75 & $3 \times 20$ & 0.6 & 171 & $157(92 \%)$ & 81 \\
\hline Total & & & & & & & 883 & $786(89 \%)$ & 298 \\
\hline
\end{tabular}

Notes. Units of right ascension $(\alpha)$ are in hours, minutes, and seconds. Units of declination $(\delta)$ are in degrees, arcminutes, and seconds.

a The "M32_1" mask was originally named "M32" at the time of submission of the mask design.

${ }^{b}$ Buckling of the M32_3 DEIMOS multislit mask at the time of observation adversely affected $\approx 25 \%$ of its slitlets. 


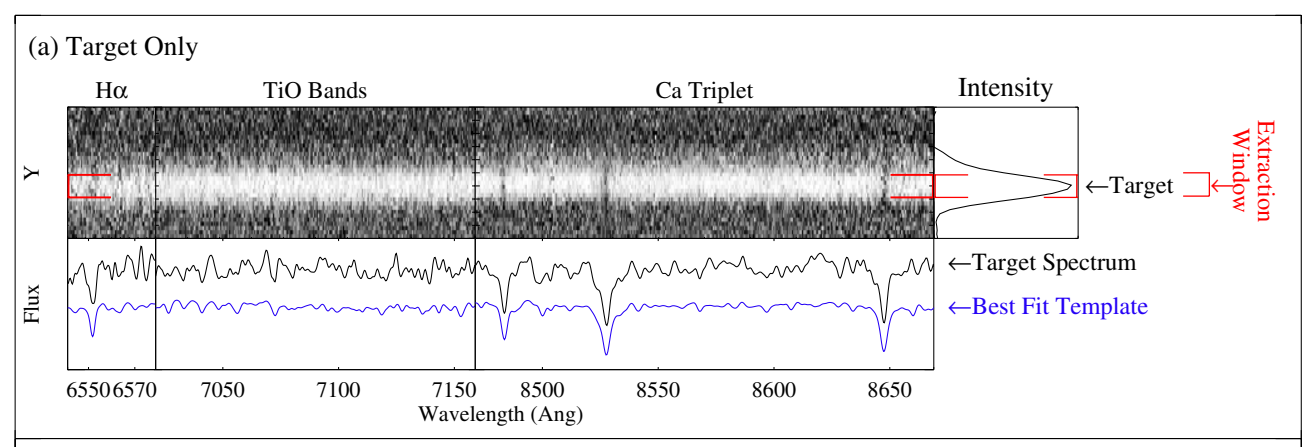

(b) Target + Off-Target Serendip (only serendip 1D spectrum shown)
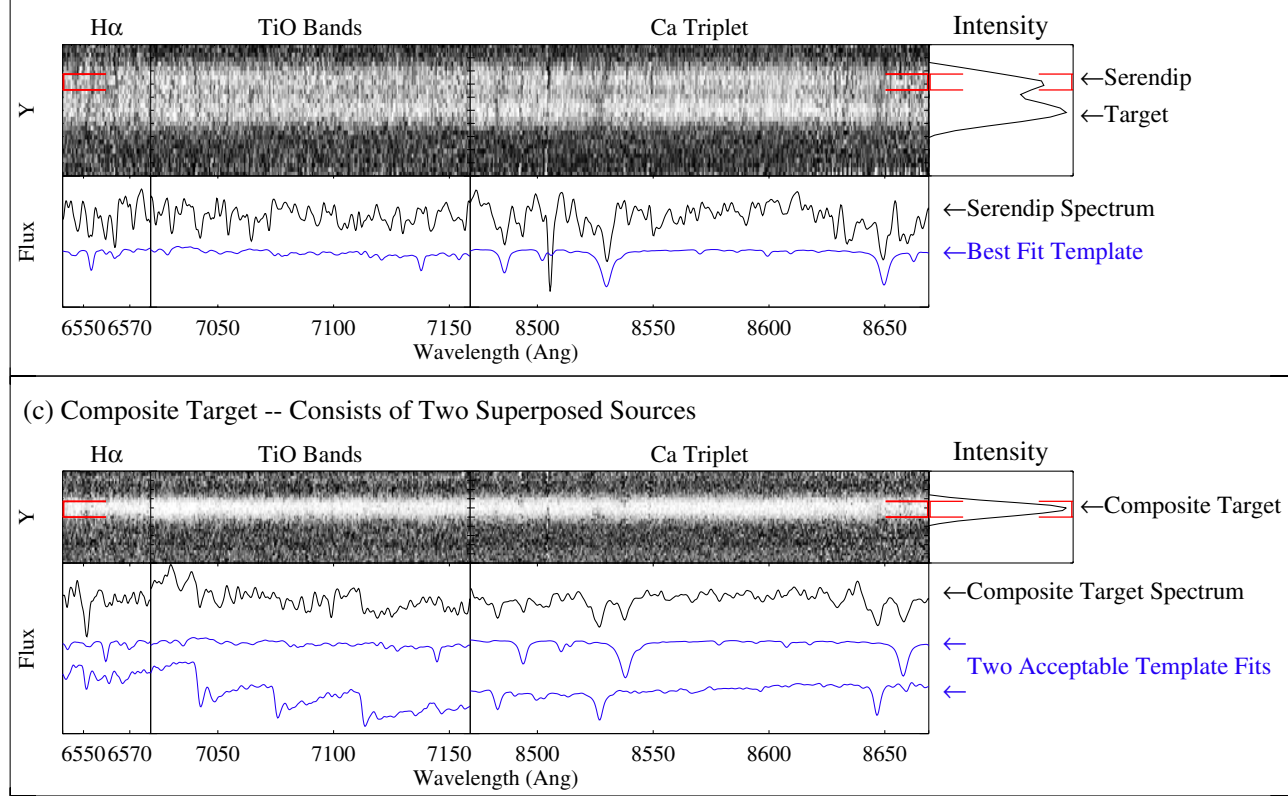

Figure 4. Examples of target stars and serendipitous sources (serendips). Each panel (a-c) shows a slitlet's two-dimensional spectrum (top left) where the wavelength axis $x$ runs horizontally (zoomed in to highlight specific spectral features), and the spatial axis $y$ (position along the slitlet) runs vertically. The horizontal streak(s) within each two-dimensional spectrum is the stellar continuum from RGB candidate(s). The spatial location of the continuum is determined by collapsing the two-dimensional spectrum in the wavelength direction and identifying peaks in the intensity profile (top right, black). One-dimensional stellar spectra (bottom, black) are extracted from the two-dimensional spectrum using a small spatial extraction window (top, red) that straddles the continuum of the desired star. The LOS velocity of a star is measured by cross-correlating Doppler shifted stellar templates with the one-dimensional stellar spectrum until a best match is found (bottom, blue). Note that the best-fit Doppler shifted stellar template (blue) is offset from the stellar spectrum (black) for the purpose of illustration. (a) Example of a slitlet intersecting a single star (i.e., the target star). (b) Example of a slitlet intersecting two spatially resolved stars (i.e., the target star and an off-target serendip). The one-dimensional spectrum and best-fit stellar template shown correspond to the serendip. (c) Example of a slitlet intersecting two spatially blended stars (i.e., the target star and an on-target serendip). The presence of multiple stars is revealed by the two sets of absorption features in the one-dimensional spectrum.

(A color version of this figure is available in the online journal.)

in reduced $\chi^{2}$ space. The ten best-fit templates, LOS velocities, reduced- $\chi^{2}$ values and cross-correlation errors are reported. The stellar templates used in the cross-correlation analysis cover a wide range of stellar types, F8 to M8 giants, subgiants and dwarf stars, and metallicities, $[\mathrm{Fe} / \mathrm{H}]=-2.12$ to +0.11 (Simon $\&$ Geha 2007). The observing setup for the templates is nearly identical to that discussed in Section 2.1.5, with the exception that template stars are observed with 0.7 wide slitlets, the minimum slit length is set to $4^{\prime \prime}$ (to allow for adequate sky subtraction), and the template stars are trailed across the slit.

$A$-band telluric corrections and heliocentric corrections are calculated and applied to the measured LOS velocities. The $A$-band telluric corrections, which account for velocity errors associated with the slight mis-centering of a star in a slit, are determined using the method discussed in Sohn et al. (2007).

LOS velocity errors are determined for each star by scaling the cross-correlation based velocity error using duplicate radial velocity measurements of stars (K. Gilbert 2011, private communication). The average LOS velocity error for M32 RGB for each star $\Delta v$ is estimated to be:

$$
\Delta v=\sqrt{\left(1.85 \times \Delta v_{\mathrm{cc}}\right)^{2}+2.2^{2}}
$$

where $\Delta v_{\mathrm{cc}}$ is the cross-correlation based error and $2.2 \mathrm{~km} \mathrm{~s}^{-1}$ is the systematic velocity error as determined by Simon \& Geha (2007). The scale factor 1.85 is determined from duplicate radial velocity measurements of stars. The average LOS velocity error for M32 RGB stars is $4 \mathrm{~km} \mathrm{~s}^{-1}$.

\subsubsection{Quality Assessment}

Each two-dimensional spectrum, one-dimensional spectrum, and corresponding Doppler shifted template match, are visually inspected in zspec and assigned a quality code $Q$ based on the reliability of the fit. This process allows the user to judge the quality of a spectrum and reject instrumental failures and poor quality spectra. Velocity measurements based on two or more strong spectral features are assigned $Q=4$ (82\% of targets). Velocity measurements based on one strong feature 
plus additional marginal features are assigned $Q=3(6 \%$ of targets). Spectra that contain no strong features, low $\mathrm{S} / \mathrm{N}$, and/or instrumental failures are assigned $Q=2$ ( $8 \%$ of targets). For cases in which zspec did not return an accurate velocity measurement, but visual inspection of the one-dimensional spectrum showed an obvious velocity shift, the velocity is manually marked and assigned $Q=1$ ( $<1 \%$ of targets). Foreground stars used for the purpose of alignment are assigned $Q=-1$ (3\% of targets). Additional details on this quality code assignment can be found in Guhathakurta et al. (2006).

\subsubsection{Serendipitous Sources}

Upon visual inspection of the one-dimensional and twodimensional spectra during the quality assessment phase outlined in Section 2.1.8, some fraction of the slits clearly show that the full length of the slitlet intersects more than one star: the target star and one or more serendipitously detected stars, known hereafter as a serendips. These detections occur frequently in our target region due to the severe crowding and blending in the CFHT MegaCam data. Our de-blending exercises for target selection (see Section 2.1.3) are good only to the 0.8 seeing limit of the CFHT MegaCam data. The better angular resolution of the Keck/DEIMOS spectroscopic data $\left(\sim 00^{\prime} 6\right)$ allows for the spatial resolution of stars that cannot be resolved in the CFHT MegaCam data.

Serendips are detected via one of two methods: through continuum detections that are offset from the primary target in the spatial direction (referred to as off-target serendips), or by the detection of spectral features that are offset from the primary target in the spectral direction (referred to as on-target serendips). In a couple of rare cases, we detect stars using a combination of the two methods (referred to as off-target superimposed serendips). We discuss the details of these detection methods in turn.

Off-target serendips are visually identified as additional brightness peaks in the spatial intensity profile that are offset from the target and spatially coincident with the spectral continuum in the two-dimensional spectrum (see Figure 4(b)). There are cases where the spectral continuum is distinct from the target and instances where it is partially blended with the target (i.e., the spatial intensity profile shows a peak with an asymmetric wing). Once the location of the off-target serendip has been visually identified in the spatial intensity profile, an extraction window is manually placed on the two-dimensional spectrum and the one-dimensional spectrum is extracted. The reduction process then proceeds as outlined in Sections 2.1.7 and 2.1.8. LOS velocities are measured for 244 off-target serendips over the five masks.

On-target serendips are cases where the spatial intensity profile shows only a single peak (i.e., looks like one star) but where 2 sets of spectral absorption features are evident (Ca II, TiO, etc., see Figure 4(c)) in the one-dimensional spectrum. We require a minimum of two pronounced absorption features at the same LOS velocity before classification as an on-target serendip. The minimum velocity separation for which we are able to detect two distinct superimposed velocities is $\Delta v \sim 50 \mathrm{~km} \mathrm{~s}^{-1}$ (which is slightly greater than the $\sim 35 \mathrm{~km} \mathrm{~s}^{-1}$ FWHM of our instrumental resolution near the Ca II triplet). We define the fainter of the two superimposed stars as the on-target serendip. The best LOS velocity match to the on-target serendip is determined during the quality assessment phase. In cases where the ten best-fit solutions reported by zspec include a mix of fits for both the target and the on-target serendip, the LOS velocity best matching the on-target serendip is selected from the list and assigned a quality code $(Q=3$, 4). In cases where the solutions reported by zspec do not show any good matches to the on-target serendip, the best fit is determined by manually shifting stellar templates until a good match is found; these fits are assigned $Q=1$. Once the LOS velocity has been determined, a heliocentric and an average telluric correction is applied, and the LOS velocity error is calculated. LOS velocities are measured for 52 on-target serendips over the five Keck/DEIMOS multislit masks.

Off-target superimposed serendips are a combination of the two categories discussed above. They consist of cases where a continuum that is offset from the primary target turns out to be two perfectly blended serendips at the same spatial location. The one-dimensional spectrum of the off-target superimposed serendip is extracted via the method outlined for off-target serendips. The zspec software is then run on the one-dimensional spectrum, and analysis proceeds as for on-target serendips. We find two such instances of off-target superimposed serendips over the five Keck/DEIMOS multislit masks.

LOS velocities are measured for a total number of 298 serendips in all three classes. The number of serendip LOS velocities measured per mask is summarized in Table 2.

\subsubsection{Maximum Likelihood Analysis of the Line-of-sight Velocities}

A subsample of the measured target and serendip LOS velocities is selected for further kinematical analysis based on their probability of M32 membership, as determined by sky position. Stars with a probability $P \geqslant 5 \%$ (see Figure 1) are selected as potential M32 candidates. This reduces the stellar sample from 1084 to 482 stars and corresponds to a radial range of $0.2-1.4 \mathrm{kpc}$. In order to determine if strong velocity and/or velocity dispersion gradients are present along M32's major and minor axes, the subsample of stars is further divided into eight subregions: four quadrants divided into two probability ranges. The four quadrants, outlined in Figure 5, are centered on M32's axes as follows: north-north-west (NNW) major-axis $\left(-65^{\circ} \leqslant \phi \leqslant 25^{\circ}\right.$, spanning $0.2-0.9 \mathrm{kpc}$ ), south-south-east (SSE) major-axis $\left(115^{\circ} \leqslant \phi \leqslant 205^{\circ}\right.$, spanning $\left.0.3-1.4 \mathrm{kpc}\right)$, west-south-west (WSW) minor-axis $\left(205^{\circ} \leqslant \phi \leqslant 295^{\circ}\right.$, spanning $0.2-1.0 \mathrm{kpc}$ ), and east-north-east (ENE) minor-axis $\left(25^{\circ} \leqslant \phi \leqslant 115^{\circ}\right.$, spanning $\left.0.3-1.0 \mathrm{kpc}\right)$. The lack of symmetry between the contours in M32's SSE and NNW quadrants is due to differing amounts of M31 light contamination; the M31 contamination is significantly worse in M32's NNW region, resulting in contours that extend further out on the SSE side of the galaxy. This effect can also be seen along the minor-axis of the galaxy where the contours extend further out on the ENE side of the galaxy. The two probability ranges are defined so that each subregion contains a reasonable number of M32 stars: an inner region defined by $P \geqslant 50 \%(0.2 \lesssim a \lesssim 0.6 \mathrm{kpc})$, and an outer region defined by $5 \% \leqslant P<50 \%(0.5 \lesssim a \lesssim 1.4 \mathrm{kpc})$. These eight subregions are illustrated in Figure 5.

We perform maximum likelihood fits of Gaussians to the LOS velocity distribution of stars in the M32 region (all stars with $P \geqslant 5 \%$ ) as discussed in the following paragraphs. While the true shape of the velocity structural components in these regions may differ from pure Gaussians, the use of such models seems appropriate given their ability to characterize the mean velocity and velocity dispersion, the small number of velocity points being assessed, and the absence of any definite physical model. The individual stellar velocity errors are not included in the maximum likelihood analyses, since these errors 


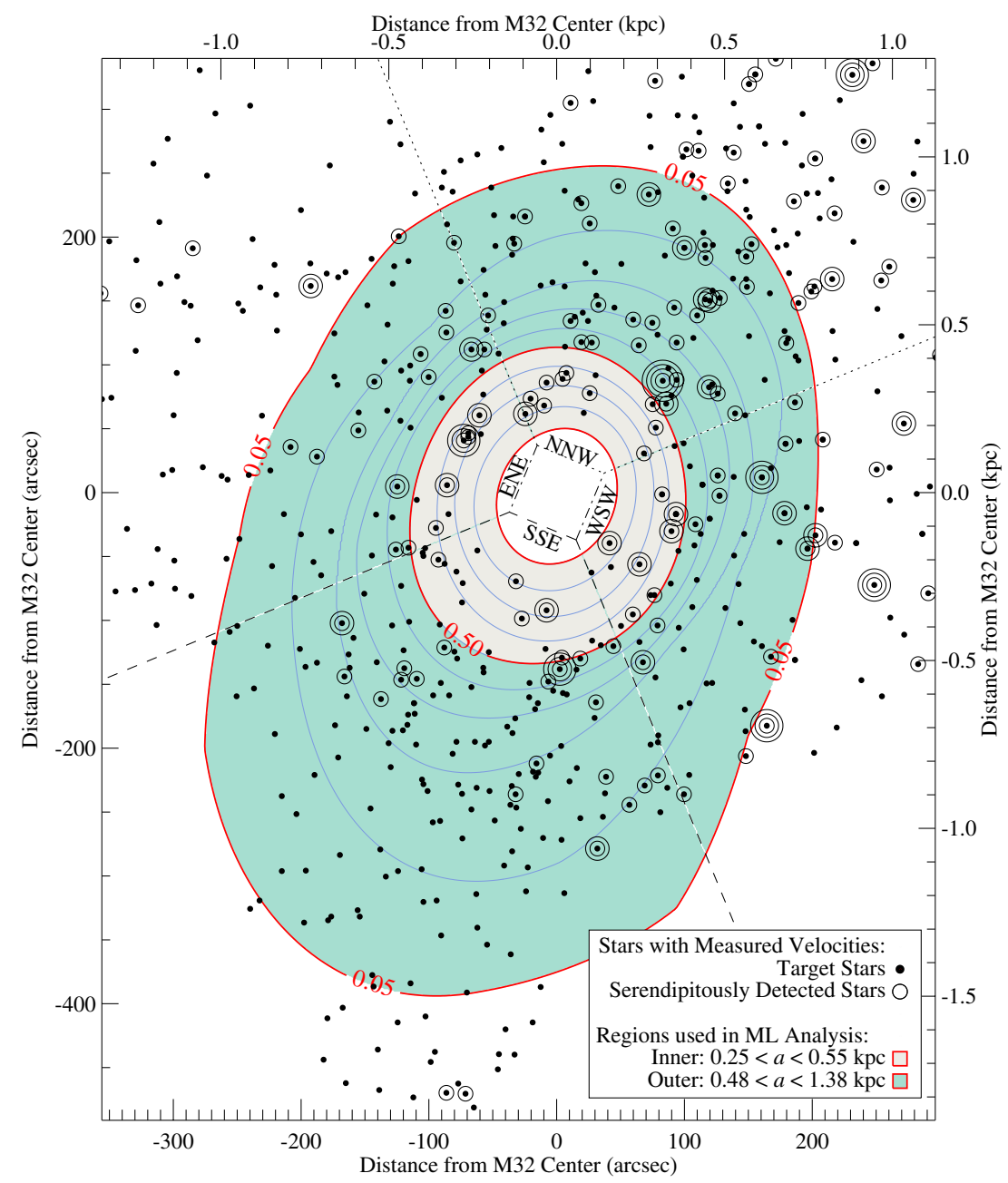

Figure 5. A subsample of targets (closed circle) and serendips (open circle) used for kinematical analysis. Concentric open circles denote multiple serendipitous detections at a given location. The contours shown outline M32's predicted fractional I-band light contribution relative to M31's inner spheroid and disk. For the purpose of kinematical analysis, the M32 region is divided into four quadrants: NNW major-axis, SSE major-axis, WSW minor-axis, and ENE minor-axis. In order to determine if strong velocity gradients are present, each quadrant is further divided into three subregions based on the probability, $P$, of M32 membership (derived from two-dimensional surface brightness profiles of M31 and M32). The subregions for each quadrant are defined as follows: inner $\equiv 50 \% \leqslant P \leqslant 90 \%$ (corresponding to a semi-major axis distance of $0.25 \lesssim a \lesssim 0.55 \mathrm{kpc}$ ), and outer $\equiv 5 \% \leqslant P<50 \%(0.48 \lesssim a \lesssim 1.38 \mathrm{kpc}$ ). These subregions are chosen (somewhat arbitrarily) so that the inner and outer subregions contain a reasonable number of M32 stars. Maximum likelihood fits of Gaussians to the LOS stellar velocities in each of these subregions are shown in Figure 6.

(A color version of this figure is available in the online journal.)

$\left(\sim 4 \mathrm{~km} \mathrm{~s}^{-1}\right)$ are much smaller than the velocity dispersion of any structural component in any of these regions, and therefore contribute insignificantly to the maximum likelihood errors. The Gaussian fits to the M32-like resolved stellar LOS velocities in each region and corresponding 68\% and $90 \%$ confidence limit errors are summarized in Table 3 and discussed below in turn.

Figure 6 shows maximum likelihood fits of the sums of Gaussians to the LOS velocity distribution of stars for the entire M32 region (all stars with $P \geqslant 5 \%$ ). The observed LOS velocity distribution is well fit by the sum of three Gaussians (red curve). The narrow Gaussian centered at $v=-196.9 \pm 3.0 \mathrm{~km} \mathrm{~s}^{-1}$, with a width of $\sigma=29.9 \pm 2.9 \mathrm{~km} \mathrm{~s}^{-1}$ (solid black curve) represents $35.2_{-3.6}^{+2.8} \%$ of the stars in the region and is consistent with the systemic velocity of M32 ( $v_{\text {sys }}^{\mathrm{M} 32}=-200 \mathrm{~km} \mathrm{~s}^{-1}$, Falco et al. 1999). The two additional populations seen in this region are well fit by a broad Gaussian centered at $v=-350.9 \mathrm{~km} \mathrm{~s}^{-1}$, with a width of $\sigma=153.0 \mathrm{~km} \mathrm{~s}^{-1}$ representing $41.9 \%$ of the stars, and a narrow Gaussian centered at $v=-386.9 \mathrm{~km} \mathrm{~s}^{-1}$, with a width of $\sigma=35.0 \mathrm{~km} \mathrm{~s}^{-1}$ representing $22.9 \%$ of the stars; these two additional populations are consistent with the mean velocity of M31's inner spheroid, $v_{\text {sys }}^{\mathrm{M} 31} \sim-300 \mathrm{~km} \mathrm{~s}^{-1}$ (Gilbert et al. 2007), and M31's disk, $v_{\text {disk }}^{\mathrm{M} 31} \sim-400 \mathrm{~km} \mathrm{~s}^{-1}$, respectively.

Figure 7 shows maximum likelihood fits of the sums of Gaussians to the LOS velocity distribution of stars to each subregion located along M32's major-axis and minor-axis, respectively. In each subregion, the two Gaussians that represent M31's inner spheroid and disk (determined in the global fit to the M32 region) are held fixed while the best-fit Gaussian parameters to M32 members are searched. The mean velocity $v$, velocity dispersion $\sigma$, and fraction $N_{\mathrm{M} 32} / N_{\text {tot }}$ of M32 stars and the corresponding $68 \%$ and $90 \%$ confidence limits for each subregion are listed in Table 3.

We compare the M32/M31 fraction predicted by our 2D surface brightness models for the two galaxies to the fraction measured from the fit to the LOS velocity distribution in each subregion. While the two sets of fractions follow similar trends across the different subregions, the surface brightness-based predictions of the M32 fraction tend to be systematically lower than the fractions measured from the LOS velocity distribution 
Table 3

Maximum Likelihood Gaussian Best-fit Parameters to M32-like Resolved Stellar Velocities

\begin{tabular}{|c|c|c|c|c|c|c|c|c|c|c|}
\hline \multirow{2}{*}{$\begin{array}{c}\bar{r}^{\mathrm{a}} \\
\text { Confidence Limits }\end{array}$} & \multirow[t]{2}{*}{$\operatorname{rms}(r)^{\mathrm{b}}$} & \multirow[t]{2}{*}{$v^{\mathrm{c}}$} & \multicolumn{2}{|c|}{$\Delta v^{\mathrm{d}}$} & \multirow[t]{2}{*}{$\sigma^{\mathrm{e}}$} & \multicolumn{2}{|c|}{$\Delta \sigma^{\mathrm{f}}$} & \multirow[t]{2}{*}{$N_{\mathrm{M} 32} / N_{\mathrm{tot}^{\mathrm{g}}}^{\mathrm{g}}$} & \multicolumn{2}{|c|}{$\Delta N_{\mathrm{M} 32} / N_{\mathrm{tot}}^{\mathrm{h}}$} \\
\hline & & & $(68 \%)$ & $(90 \%)$ & & $(68 \%)$ & $(90 \%)$ & & $(68 \%)$ & $(90 \%)$ \\
\hline \multicolumn{11}{|c|}{ M32, All Quadrants, Inner + Outer } \\
\hline $174^{\prime \prime} .2$ & $65^{\prime \prime} 0$ & -196.9 & \pm 3.0 & $\begin{array}{l}+5.0 \\
-4.9\end{array}$ & 29.9 & $\begin{array}{l}+3.1 \\
-2.9\end{array}$ & $\begin{array}{l}+5.2 \\
-4.6\end{array}$ & 0.352 & \pm 0.028 & $\begin{array}{l}+0.047 \\
-0.046\end{array}$ \\
\hline \multicolumn{11}{|l|}{ Major Axis: } \\
\hline \multicolumn{11}{|c|}{ M32, NNW Quadrant, Inner } \\
\hline$-95^{\prime \prime} .4$ & $11^{\prime \prime} .9$ & -198.7 & $\begin{array}{l}+6.4 \\
-6.5\end{array}$ & $\begin{array}{l}+10.8 \\
-11.4\end{array}$ & 24.2 & $\begin{array}{l}+6.0 \\
-4.4\end{array}$ & $\begin{array}{l}+11.8 \\
-7.0\end{array}$ & 0.742 & $\begin{array}{l}+0.092 \\
-0.110\end{array}$ & $\begin{array}{l}+0.140 \\
-0.189\end{array}$ \\
\hline \multicolumn{11}{|c|}{ M32, NNW Quadrant, Outer } \\
\hline$-201^{\prime \prime} .2$ & $43 " .1$ & -226.4 & $\begin{array}{l}+11.0 \\
-11.4\end{array}$ & $\begin{array}{l}+18.4 \\
-20.0\end{array}$ & 34.1 & $\begin{array}{l}+9.9 \\
-7.4\end{array}$ & $\begin{array}{l}+19.3 \\
-11.8\end{array}$ & 0.196 & $\begin{array}{l}+0.055 \\
-0.052\end{array}$ & $\begin{array}{l}+0.094 \\
-0.084\end{array}$ \\
\hline \multicolumn{11}{|c|}{ M32, SSE Quadrant, Inner } \\
\hline $123^{\prime \prime} .8$ & $23 " .4$ & -186.3 & \pm 9.0 & $\begin{array}{l}+15.2 \\
-15.4\end{array}$ & 31.2 & $\begin{array}{l}+7.2 \\
-5.5\end{array}$ & $\begin{array}{l}+13.5 \\
-8.5\end{array}$ & 0.663 & $\begin{array}{l}+0.107 \\
-0.121\end{array}$ & $\begin{array}{l}+0.165 \\
-0.204\end{array}$ \\
\hline \multicolumn{11}{|c|}{ M32, SSE Quadrant, Outer } \\
\hline $225^{\prime \prime} 6$ & $53 \prime .5$ & -191.0 & $\begin{array}{l}+7.2 \\
-7.1\end{array}$ & \pm 11.9 & 36.2 & $\begin{array}{l}+6.2 \\
-5.4\end{array}$ & $\begin{array}{l}+11.0 \\
-8.8\end{array}$ & 0.311 & \pm 0.051 & $\begin{array}{l}+0.080 \\
-0.084\end{array}$ \\
\hline \multicolumn{11}{|c|}{ Minor Axis: } \\
\hline \multicolumn{11}{|c|}{ M32, ENE Quadrant, Inner } \\
\hline-106.2 & $5^{\prime \prime} .1$ & -184.4 & $\begin{array}{l}+7.0 \\
-8.4\end{array}$ & $\begin{array}{l}+11.7 \\
-14.3\end{array}$ & 26.2 & $\begin{array}{l}+7.8 \\
-5.2\end{array}$ & $\begin{array}{l}+13.5 \\
-7.6\end{array}$ & 0.788 & $\begin{array}{l}+0.094 \\
-0.113\end{array}$ & $\begin{array}{l}+0.139 \\
-0.195\end{array}$ \\
\hline \multicolumn{11}{|c|}{ M32, ENE Quadrant, Outer } \\
\hline-142 .' 6 & 30.2 & -204.6 & $\begin{array}{l}+6.9 \\
-7.0\end{array}$ & $\begin{array}{l}+11.4 \\
-11.8\end{array}$ & 26.0 & $\begin{array}{l}+6.3 \\
-5.9\end{array}$ & $\begin{array}{l}+11.0 \\
-9.0\end{array}$ & 0.401 & \pm 0.080 & $\begin{array}{l}+0.131 \\
-0.130\end{array}$ \\
\hline \multicolumn{11}{|c|}{ M32, WSW Quadrant, Inner } \\
\hline $\begin{array}{r}119^{\prime \prime} 3 \\
\end{array}$ & $21^{\prime \prime} .4$ & -194.6 & \pm 4.2 & $\begin{array}{l}+7.1 \\
-7.0\end{array}$ & 18.1 & $\begin{array}{l}+3.3 \\
-2.7\end{array}$ & $\begin{array}{l}+6.0 \\
-4.3\end{array}$ & 0.724 & $\begin{array}{l}+0.083 \\
-0.096\end{array}$ & $\begin{array}{l}+0.128 \\
-0.163\end{array}$ \\
\hline \multicolumn{11}{|c|}{ M32, WSW Quadrant, Outer } \\
\hline $156^{\prime \prime} \cdot 3$ & $28 . .9$ & -179.2 & $\begin{array}{l}+11.7 \\
-11.3 \\
\end{array}$ & $\begin{array}{l}+20.2 \\
-23.0 \\
\end{array}$ & 39.0 & $\begin{array}{l}+9.7 \\
-7.8 \\
\end{array}$ & $\begin{array}{l}+18.7 \\
-21.8 \\
\end{array}$ & 0.297 & $\begin{array}{l}+0.072 \\
-0.070 \\
\end{array}$ & $\begin{array}{l}+0.120 \\
-0.113 \\
\end{array}$ \\
\hline
\end{tabular}

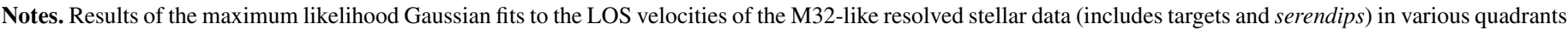
located within M32's 5\% predicted fractional light contribution contour (see Figure 5).

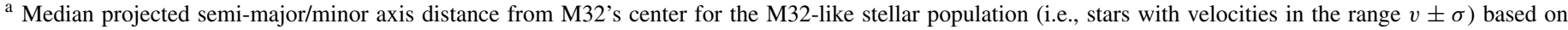

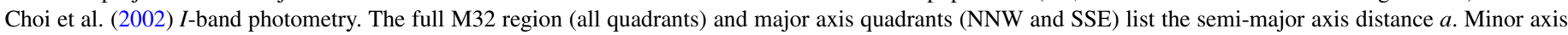
quadrants (ENE and WSW) list the semi-minor axis distance $q a$.

${ }^{b}$ Root-mean-square of the semi-major/minor axis distance.

c Best-fit heliocentric LOS velocity.

${ }^{\mathrm{d}}$ Error in best-fit heliocentric LOS velocity (68\% and $90 \%$ confidence limits).

e Best-fit velocity dispersion.

${ }^{\mathrm{f}}$ Error in best-fit velocity dispersion (68\% and $90 \%$ confidence limits).

$\mathrm{g}$ Best-fit fraction of M32 stars based on kinematics.

${ }^{\mathrm{h}}$ Error in best-fit fraction of M32 stars in the sample (68\% and $90 \%$ confidence limits).

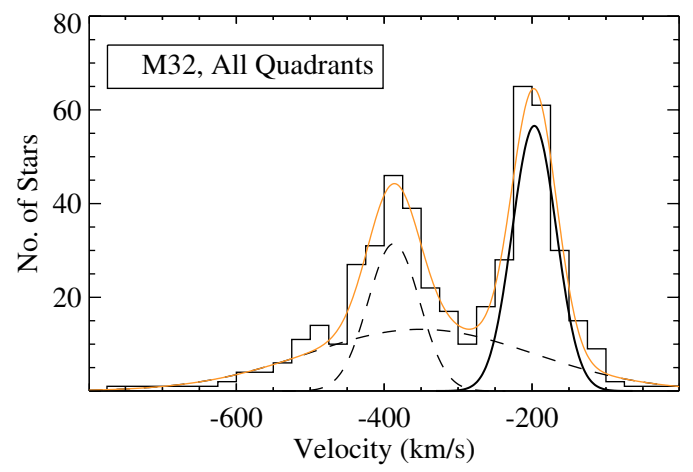

Figure 6. Velocity histogram and maximum likelihood Gaussian fits to the LOS velocities of resolved stars located within M32's 5\% predicted fraction light contribution contour (see Figure 5). The solid black curve shows the fit that is consistent with M32's systemic velocity $\left(v_{\mathrm{sys}}^{\mathrm{M} 32}=-200 \mathrm{~km} \mathrm{~s}^{-1}\right.$; Falco et al. 1999). The dashed black curves have mean velocities and velocity dispersions consistent with a cold rotating component (M31's disk), with a hot non-rotating component (M31's inner spheroid). The sum of the Gaussian fits is shown as a solid orange curve. The fits to the M31-like stellar population are held fixed during the analysis of the quadrants surrounding M32.

(A color version of this figure is available in the online journal.)

analysis (but by only $\sim 1 \sigma$ on average). In any case, this slight discrepancy does not affect any of the results of this paper. We only use the surface brightness based fraction predictions to define the boundaries of the subregions in which to carry out the kinematical fits, and these boundaries are fairly arbitrarily defined in any case.

In principle, it might be possible to use the combined information from kinematics and imaging to derive better constraints on the surface brightness profile of M32 at large radii. For example, the fact that the kinematically-determined M32 fractions are somewhat higher than those predicted by our surface brightness model could suggest that the adopted surface brightness profile for M32 is too steep. However, there also exist alternative interpretations for this finding. First, M31's disk surface brightness is not uniform across the M32 region and may well depart from our idealized 2D surface brightness model. Second, our spectroscopic target selection tends to bias the kinematical sample against high surface brightness/crowded patches in M31's disk, resulting in a bias toward higher M32 fractions. Third, the translation from integrated $V$-band surface brightness to RGB star count surface density is likely not the same for M32, M31's inner spheroid, and M31's disk, and may well vary with radius within the two galaxies. Finally, there are uncertainties in our 2D surface brightness models for the two galaxies associated with $I$ - to $V$-band conversion of the surface brightness measurements. Given these complexities, we have not attempted to use the kinematical measurements to better constrain the M32 surface brightness profile.

Our kinematically based measurement of the M31 contamination fraction is relevant for M32 stellar population studies. 
Table 4

Keck/DEIMOS Long-slit Exposures

\begin{tabular}{|c|c|c|c|c|c|c|c|}
\hline $\begin{array}{l}\text { Mask } \\
\text { Name }\end{array}$ & $\begin{array}{c}\text { Observation } \\
\text { Date }\end{array}$ & $\begin{array}{c}\alpha \\
(\mathrm{J} 2000.0)\end{array}$ & $\begin{array}{c}\delta \\
(\mathrm{J} 2000.0)\end{array}$ & $\begin{array}{l}\text { P.A. } \\
\text { (deg) }\end{array}$ & $\begin{array}{l}t_{\exp } \\
(\mathrm{m})\end{array}$ & $\begin{array}{l}\text { Seeing } \\
\text { FWHM }\end{array}$ & $\begin{array}{c}\text { Slit } \\
\text { Width }\end{array}$ \\
\hline Major_1 & 2007 Nov 14 & 004241.87 & +405157.2 & 160 & 3 & 0.5 & $1^{\prime \prime} 0$ \\
\hline Major_2 & 2008 Nov 14 & 004241.87 & +405157.2 & 160 & 3 & 0.5 & $1^{\prime \prime} 0$ \\
\hline Major_3 & 2008 Oct 1 & 004241.87 & +405157.2 & 160 & 5 & $\sim 1^{\prime \prime} .0$ & $1^{\prime \prime} 0$ \\
\hline Major_4 & 2008 Oct 1 & 004241.87 & +405157.2 & 160 & 5 & $\sim 1^{\prime \prime} .0$ & $1^{\prime \prime} 0$ \\
\hline Major_5 & 2008 Nov 24 & 004241.87 & +405157.2 & -20 & 5 & $1^{\prime \prime} .3$ & 0.8 \\
\hline Minor_1 & 2008 Nov 24 & 004241.87 & +405157.2 & 70 & 5 & $1^{\prime \prime} 3$ & 0.8 \\
\hline Minor_2 & 2008 Nov 24 & 004241.87 & +405157.2 & 70 & 5 & $1^{\prime \prime} .3$ & 0.8 \\
\hline
\end{tabular}

Notes. The units of right ascension $(\alpha)$ are in hours, minutes, and seconds. The units of declination $(\delta)$ are in degrees, arcminutes, and seconds.

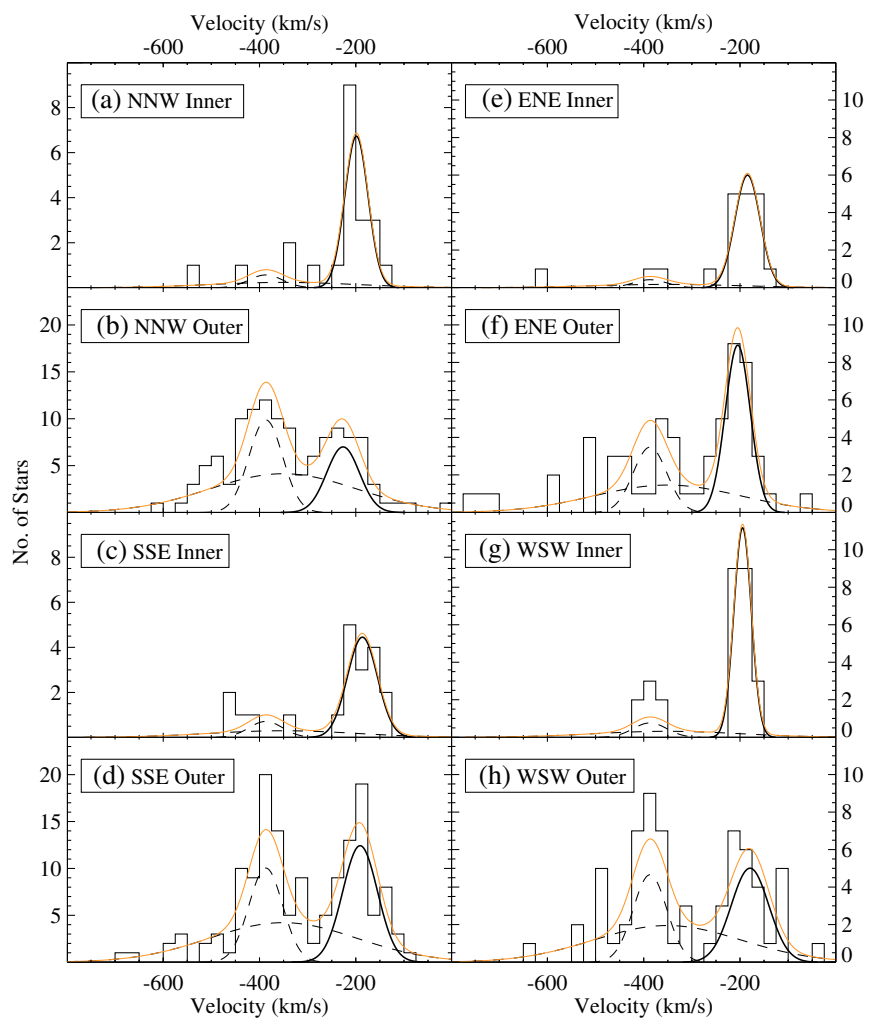

Figure 7. Velocity histograms and maximum likelihood Gaussian fits to the LOS velocities of resolved stars along the major and minor axes of M32. Solid black curves show fits that are consistent with M32's systemic velocity $\left(v_{\text {sys }}^{\text {M32 }}=-200 \mathrm{~km} \mathrm{~s}^{-1}\right.$; Falco et al. 1999). Dashed black curves (M31 components) have fixed ratios, velocities, and velocity dispersions that are based on a global fit to the M32 region (Figure 6). The sum of the Gaussian fits is shown as a solid orange curve. Panels (a)-(h) show fits to the subregions outlined in Figure 5: (a) the inner region along M32's NNW semi-major axis, (b) the outer region along M32's NNW semi-major axis, (c) like (a) but for SSE major axis, (d) like (b) but for SSE major axis, (e) like (a) but for ENE semi-minor axis, and (f) like (b) but for ENE semi-minor axis, (g) like (a) but for the WSW minor axis, and (h) like (b) but for the WSW minor axis.

(A color version of this figure is available in the online journal.)

For example, in the recent Monachesi et al. (2011) analysis of M32's star-formation history from deep HST ACS/HRC CMDs, contamination by M31 stars is statistically accounted for by using a control field whose location was chosen on the basis of the shape/orientation of M31's disk isophotes. Our kinematical analysis provides an independent measurement of the M31 contamination fraction in the region of their study, albeit averaged over a larger area than the narrow HST ACS/HRC field.

\subsection{Integrated Light Spectroscopy}

We begin this subsection by describing observations, and end with measurements of the line-of-sight velocity distribution (LOSVD) along M32's major and minor axes from the integrated light data.

\subsubsection{Observations}

Eight longslit exposures centered on M32 were obtained with Keck/DEIMOS between 2007 and 2008 November. Six of the longslits were aligned with M32's major-axis $\left(\phi=160^{\circ}\right)$, and two were aligned with M32's minor-axis $\left(\phi=70^{\circ}\right)$. Each longslit mask is $\sim 16^{\prime}$ long and designed with a series of 12 slits separated by small bridges in order to ensure the structural integrity of the mask design. The observing setup for the longslit exposures is identical to that used for the multislit mask observations discussed in Section 2.1.5, except that slit widths of $0{ }^{\prime} .8$ and $1^{\prime \prime} .0$ were used. The average seeing for these observations was 1 .'0, yielding an average spectral resolution of 3.9 pixels $=1.3 \AA$ for the $1^{\prime \prime} .0$ slit width. The observing details for the longslit observations are summarized in Table 4.

\subsubsection{Data Reduction}

Processing of the eight Keck/DEIMOS longslit masks from rectification through wavelength calibration is identical to the reduction procedure used for the Keck/DEIMOS multislit masks: the two-dimensional spectra are rectified, flat-field corrected, fringe corrected, and wavelength calibrated using the spec $2 d$ reduction pipeline (see Section 2.1.6).

One-dimensional spectra are extracted at increasing spatial intervals from the center of M32 along the two-dimensional spectrum. The location of M32's center is determined by fitting a Moffat profile+1st order polynomial to the intensity profile of M32 (obtained by collapsing the two-dimensional spectrum in the wavelength direction); the fractional spatial pixel location that corresponds to the peak of the intensity profile fit is defined as M32's center. This information is used to convert the pixel positions along the two-dimensional spectrum into a distance from M32. One-dimensional spectra are then extracted using boxcar extraction windows with widths ranging from $1^{\prime \prime}$ to $5^{\prime \prime}$ (the actual size of the extraction window is determined by the $\mathrm{S} / \mathrm{N}$ of the region being extracted), and the Poisson errors are calculated. The one-dimensional spectra are rebinned logarithmically in the wavelength direction into bins with $13.8 \mathrm{~km} \mathrm{~s}^{-1}$ pixel $^{-1}$.

Next, sky subtraction is performed on the one-dimensional spectra. Extreme care is taken to properly subtract the light from 


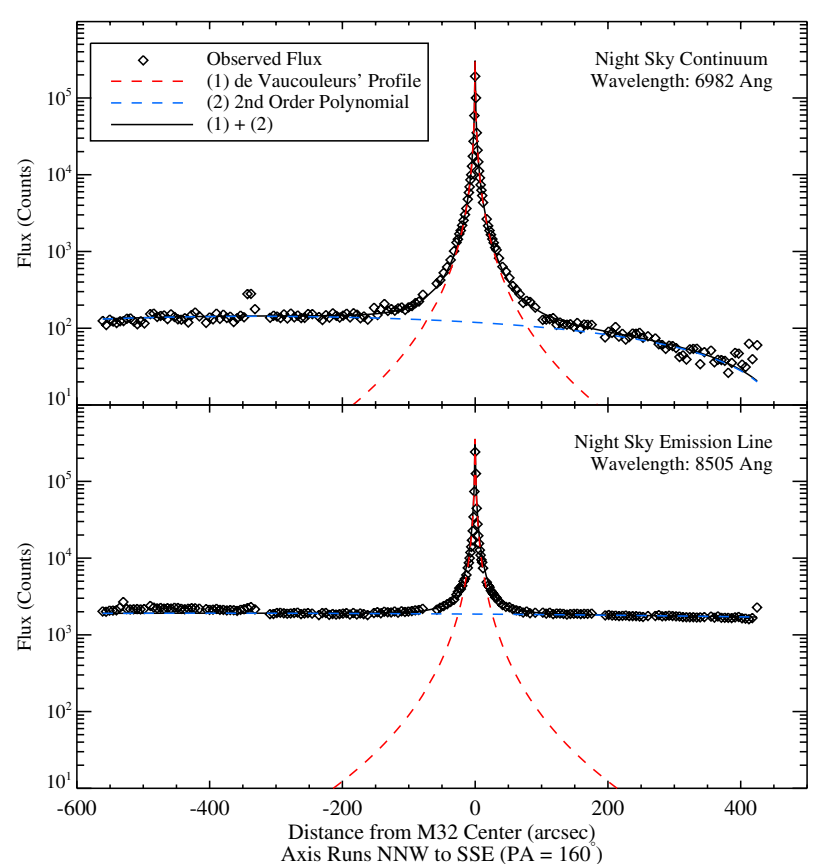

Figure 8. An example of sky subtraction and the night sky variations in M32's integrated light profile. The plot shows light intensity (black diamonds) versus distance from M32's center in two wavelength bins. The red dashed line provides a fit to M32's light by normalizing a de Vaucouleurs' profile, with $r_{I}^{\text {eff }}=29^{\prime \prime}$ (Choi et al. 2002), to the observed intensities. The contaminating light from M31, atmospheric air glow and corrections to the DEIMOS spectrograph response function is measured by subtracting off the normalized de Vaucouleurs' profile from the observed intensities and fitting a 2nd order polynomial to the residual intensities beyond 90" from M32's center (dashed blue line). The sum of the two profiles is shown as a solid black line. Top: wavelength bin corresponding to night sky continuum. The gradient seen in the continuum (dashed blue line) is a result of light contamination from M31. Bottom: wavelength bin corresponding to a peak of a night sky emission line. The M31 gradient is not visible against the dominant night sky emission.

(A color version of this figure is available in the online journal.)

all contaminating sources, which includes M31, atmospheric air glow, and imperfect flat-fielding (resulting from differences in the illumination between the internal flat-field exposure and the on sky science exposure). Sky subtraction is performed separately for each wavelength bin. First, the intensity profile as a function of distance from M32's center is obtained for each wavelength bin. A normalized de Vaucouleurs' profile with $r_{I}^{\text {eff }}=29^{\prime \prime}$ is fit to and subtracted from each intensity profile outside the inner 5" of M32's center, where excessive flux is present (Kent 1987; Choi et al. 2002); this produces an "M32-free" intensity profile for each wavelength bin. Next, a 2nd order polynomial representing all of the contaminating light sources is fit to each "M32-free" intensity profile at positions beyond 90" from M32's center. The 2nd order polynomial fits are subtracted from the original intensity profiles, and then reassembled into one-dimensional sky-subtracted spectra. An example of the fitting and subtraction process is shown in Figure 8.

Once sky subtraction is complete, the major-axis spectra observed with the 0.8 slit width (see Table 4) are Gaussian smoothed in the spectral direction in order to match the spectral resolution of the $1^{\prime \prime} .0$ slit width spectra (since the minor-axis science spectra were all observed with the same slit width they do not require smoothing for co-addition). The smoothing length $\sigma_{\text {smooth }}$ needed for the $0^{\prime} .8$ major-axis spectra is determined to be $0.42 \AA$ using the following formula:

$$
\sigma_{\text {smooth }}=\mathrm{m} \frac{\Delta \lambda}{\Delta x} \sqrt{\frac{w_{2}^{2}-w_{1}^{2}}{8 \ln 2}},
$$

where $w_{2}=1^{\prime \prime} .0$ is the desired slit width, $w_{1}=0.8$ is the observed slit width, $m=0.606$ is the anamorphic demagnification factor at the central wavelength $7800 \AA, \Delta \lambda=0.32 \AA$ pixel $^{-1}$ is the spectral dispersion, and $\Delta x=0.1185 \mathrm{pixel}^{-1}$ is the spatial scale.

Next, the one-dimensional sky-subtracted spectra from individual exposures and original fine spatial bins are co-added, with cosmic ray rejection, into broader spatial bins. These broader bins are designed to achieve a minimum spectral $\mathrm{S} / \mathrm{N}$ ratio per pixel of 25 averaged over the Ca II triplet region. This $\mathrm{S} / \mathrm{N}$ threshold limits the radial extent of our kinematical analysis to $\leqslant 90^{\prime \prime}$ and $\leqslant 60^{\prime \prime}$ on the major and minor axes, respectively. About $15 \%$ of the individual spectra are excluded from the coadds as they were deemed, upon visual inspection, to suffer from bad subtraction of night sky emission lines and/or other systematic errors (e.g., slit edges and bad columns). Inclusion of these bad spectra in the co-adds would lead to choppier, less symmetric kinematical profiles. No co-addition is performed on spectra located at $\left|r_{\mathrm{M} 32}\right| \leqslant 2^{\prime \prime}$ as the individual spectra exceed the $\mathrm{S} / \mathrm{N}$ threshold in these bright inner regions.

\subsubsection{Measurement of Velocity, Velocity Dispersion and Higher Order Gauss-Hermite Moments}

The mean velocity $v$, velocity dispersion $\sigma$, and Gauss-Hermite moments $h 3$ through $h 6$ that best fit the observed LOSVD are determined using the pixfit software developed and described by van der Marel (1994). Briefly, the software determines the best-fit absorption line strength parameter $(\gamma)$, LOS velocity $(v)$, and velocity dispersion $(\sigma)$ for each one-dimensional integrated light science spectrum by minimizing $\chi^{2}$ between scaled, Doppler-shifted, Gaussian-broadened spectral templates and the science spectra (see Figure 9). The search for an optimal spectral template is performed separately, as discussed below. Symmetric and anti-symmetric deviations from Gaussianity are then measured by expanding the fits to include higher order Gauss-Hermite moments ( $h 3$ through $h 6$, van der Marel \& Franx 1993) for spectra with an average S/N per pixel $\gtrsim 40$.

The rms uncertainties in our best-fit parameters $x=[v, \sigma$, $h 3, h 4, h 5, h 6]$ are determined using the Poisson error in the flux of the input science spectrum. Since the Poisson-based error estimates $\Delta^{P} x$ are bound to be underestimates and do not account for systematic errors such as imperfect subtraction of night sky emission lines, template mismatch, and residual detector artifacts, we attempt to derive more realistic error estimates, $\Delta x=f \times \Delta^{P} x$, where the error scale factor $f$ is derived empirically by assuming that the true kinematical profiles in the higher $\mathrm{S} / \mathrm{N}$ outer regions of $\mathrm{M} 32\left(|r|>5^{\prime \prime}, S / N \gtrsim 40\right)$ are symmetric and smooth. The symmetry assumption implies that one half of the anti-symmetric profiles $(v, h 3$, and $h 5)$ can be point-reflected onto its other half, and that one half of the symmetric profiles ( $\sigma, h 4$, and $h 6)$ can be mirror-reflected onto its other half. The smoothness assumption implies that the folded data points $x_{\text {data }}\left(r_{i}\right)$ can be compared to a second-order polynomial $x_{\text {poly }}(r)$ that has been fit to the data. Specifically, we require that deviations of the folded profiles from the smooth 


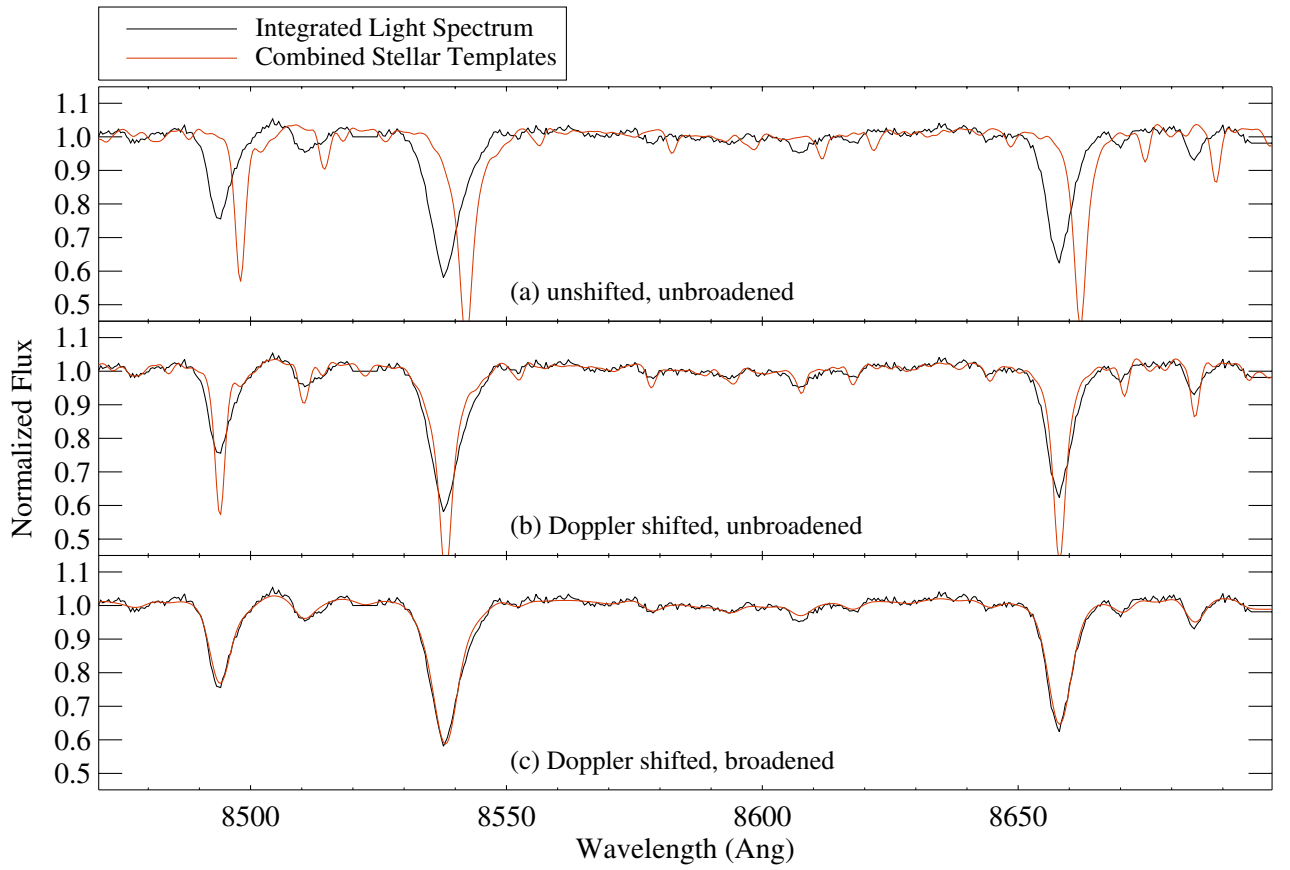

Figure 9. An illustration of the fitting of a composite stellar spectral template (red) to a $1^{\prime \prime}$ (4 pc) wide integrated light one-dimensional spectrum of M32. The composite template is constructed by optimally combining 16 stellar templates using a genetic algorithm. The three panels illustrate the steps used to measure the LOSVD of the M32 spectrum. (a) Observed M32 spectrum and an un-broadened composite stellar template (with no Doppler shift applied). (b) The Doppler velocity of the M32 spectrum is measured by shifting the un-broadened composite stellar template to match the features of the M32 spectrum. (c) The velocity dispersion of the M32 spectrum is determined by broadening the composite stellar template so that it matches the observed M32 spectrum.

(A color version of this figure is available in the online journal.)

polynomial has a $\chi^{2}$ per degree of freedom $\left(\chi_{\mathrm{DOF}}^{2}\right)$ of unity:

$$
\chi_{\mathrm{DOF}}^{2} \equiv \frac{1}{N} \sum_{i} \frac{\left[x_{\mathrm{data}}\left(r_{i}\right)-x_{\mathrm{poly}}\left(r_{i}\right)\right]^{2}}{\left[\Delta x\left(r_{i}\right)\right]^{2}}=1
$$

thereby defining the error scale factor:

$$
f^{2}=\frac{1}{N} \sum_{i} \frac{\left[x_{\mathrm{data}}\left(r_{i}\right)-x_{\mathrm{poly}}\left(r_{i}\right)\right]^{2}}{\left[\Delta^{P} x\left(r_{i}\right)\right]^{2}}
$$

Error scale factors are calculated for each of the majorand minor-axis velocity, velocity dispersion, and combined Gauss-Hermite moment profiles.

The LOSVD is analyzed over the wavelength range $8470-8700 \AA$ (i.e., the region around the Ca II triplet: 8498 , $8542,8662 \AA$ ). This wavelength range is selected to maximize the LOSVD "signal" while minimizing systematic errors. A comparative analysis of the LOSVD distribution between independent sections of the spectrum ("blue": 6500-8470 $\mathrm{\text {and }}$ "red": 8470-8900 $\AA$ ) indicates that systematic errors are relatively large in the "blue" portion. Scaling the Poisson-based errors on the LOSVD parameters to ensure "blue" versus "red" agreement overpredicts the errors by at least a factor of two relative to those derived from the full spectrum and a scaling based on the assumption of smoothness and symmetry/antisymmetry in the radial LOSVD profiles.

The spectral template used to analyze the LOSVD is constructed by combining weighted stellar templates. The choice of spectral template is of particular importance as mismatch between the science spectra and the spectral template can result in significant systematic errors (Rix \& White 1992). Given that M32 is composed of a variety of stellar types, it is not surprising that the galaxy spectra are not well fit by any single stellar spectrum. For this reason, a composite stellar template is constructed using a weighted linear superposition of stellar templates (details on the stellar templates can be found in Section 2.1.7). Since the stellar templates were observed with 0 '.7 wide slits, the templates are smoothed in the spectral direction so that they match the resolution of the science spectra; the templates are smoothed by $\sigma_{\text {smooth }}=0.50 \AA$ for comparison to the $1^{\prime \prime} .0$ resolution major-axis data, and by $\sigma_{\text {smooth }}=0.27 \AA$ for comparison to the 0.' 8 resolution minor-axis data (see Equation (3)). The optimal weights for the stellar templates are determined using a genetic algorithm (GA) as configured by Howley et al. (2008). The GA locates the global minimum in the weight parameter space by minimizing the $\chi^{2}$ between a high $\mathrm{S} / \mathrm{N}$ science spectrum and co-added weighted stellar templates, which have been Doppler shifted to match the science spectrum using cross-correlation and Gaussian smoothed using an initial velocity dispersion estimate. We assume that the velocity dispersions across the different stellar components are the same (i.e., not a function of age, stellar mass or metallicity) so that the co-added stellar templates are all smoothed with the same amount of velocity dispersion. Because template matching becomes increasingly difficult with lower $\mathrm{S} / \mathrm{N}$ spectra, the procedure is run on a single high $\mathrm{S} / \mathrm{N}$ spectrum $\left(r_{\mathrm{M} 32}=-2\right.$ ". $\left.0, \mathrm{~S} / \mathrm{N}=134\right)$ with a well measured velocity dispersion ( $\sigma=56.2 \mathrm{~km} \mathrm{~s}^{-1}$; van der Marel et al. 1994b). The intention is to use an unshifted, un-broadened version of the weighted composite stellar template to measure the velocity and velocity dispersion profiles for each science spectrum, thereby treating the populations and abundances in M32 as roughly constant with radius such that only the LOSVD, signal, and noise of each spectrum varies. Once the optimal combination of stellar templates is found, the continuum of the composite stellar template is adjusted to better match the science spectrum. 
This is achieved by fitting a 5th order polynomial to the ratio of (science spectrum)/(broadened composite stellar template), both to the blue and red sides of the spectrum separately, and multiplying the resulting polynomial by the un-broadened template to remove any low order frequency differences. The final match between the science spectrum and composite stellar template is shown in Figures 9(c) and (d).

Table 5 lists the best-fit parameters and scaled errors to the LOSVD as a function of radius. The error scale factors applied to the major-axis Poisson-based error estimates are $f_{v}=3.8$, $f_{\sigma}=2.8$, and $f_{h}=1.9$. The error scale factors applied to the minor-axis Poisson-based error estimates are $f_{v}=2.1$, $f_{\sigma}=1.9$, and $f_{h}=2.1$.

\subsection{An Integrated View of M32's Kinematics}

The results of M32's major- and minor-axis LOSVD analyses are shown in Figures 10 and 11, respectively. Our measurements push out much further than what has been observed to date. The velocity and velocity dispersion profiles are measured out to $\sim 230^{\prime \prime}$ along the major-axis and $\sim 160^{\prime \prime}$ along the minoraxis using Gaussian fits to the resolved stellar population, with integrated light measurements reaching out to $85^{\prime \prime}$ along the major-axis and $75^{\prime \prime}$ along the minor-axis. Because the integrated light velocity measurement at $85^{\prime \prime}$ on the NNW major-axis of M32 is likely affected by M31 light (pulling the velocity data point more negative), we exclude this data point from our analysis in Section 3. Measurement of the Gauss-Hermite moment profiles using integrated light extends out to $70^{\prime \prime}$ along the major-axis and 50" along the minor-axis. Our data extend well beyond the radius of $150^{\prime \prime}$ on the major-axis and $130^{\prime \prime}$ on the minor-axis where surface photometry of M32 shows a sharp upward break in the surface brightness profile and elongation and twisting of the elliptical isophotes, distortions that may be the result of tidal interaction with M31. Our profiles do not show evidence of sharp kinematical gradients across the distortion region.

A comparison of our integrated light measurements with van der Marel et al. (1994b) observations of M32's core is shown in Figures 10 and 11 (right). The profiles show good agreement, with minor differences attributable to spatial resolution, slit position and template matching. Previous studies using integrated light to measure the mass of M32's central black hole have resulted in numerous detailed velocity measurements of the core (Tonry 1987; Dressler \& Richstone 1988; van der Marel et al. 1994b; Verolme et al. 2002; van den Bosch \& de Zeeuw 2010). The most radially extensive of these studies measures a two-dimensional mean velocity, velocity dispersion, and Gauss-Hermite moments $h 3$ and $h 4$ out to $30^{\prime \prime}$ using wide field SAURON observations (Cappellari et al. 2007; van den Bosch \& de Zeeuw 2010). However, due to the large instrumental dispersion of the instrument, measurements for velocity dispersion, and $h 3$ and $h 4$ may not be very accurate (van den Bosch \& de Zeeuw 2010). Studies with higher instrumental resolution measure a velocity dispersion profile out to $13^{\prime \prime}$ along the major-axis and 22" along the minor-axis (Dressler \& Richstone 1988; van der Marel et al. 1994b), and Gauss-Hermite moments out to $8^{\prime \prime}$ along the major-axis and $22^{\prime \prime}$ along the minoraxis (van der Marel et al. 1994b).

M32's profiles appear smooth and symmetric. Our best-fit major-axis velocity profile rises steeply to a maximum observed rotational velocity of $v_{\max }=46 \mathrm{~km} \mathrm{~s}^{-1}$ at $r=1^{\prime \prime} .0(4 \mathrm{pc})$. Our best-fit minor-axis velocity profile is relatively flat, with the small cusp seen at the center of the profile resulting from a 0.25 mis-centering of the longslit during observation. The observed centrally rising velocity dispersion profile peaks at $\sigma_{\max } \approx 90 \mathrm{~km} \mathrm{~s}^{-1}$. These measurements are consistent with previous ground-based observations. Observations of the core using a narrower slit with higher spatial resolution result in higher measurements for velocity and velocity dispersion (van der Marel et al. 1997; Joseph et al. 2001).

The systemic velocity of M32 is measured as $-199.7 \pm$ $0.5 \mathrm{~km} \mathrm{~s}^{-1}$ along the major-axis and $-200.1 \pm 1.8 \mathrm{~km} \mathrm{~s}^{-1}$ along the minor-axis. These measurements are made by assuming point reflection symmetry of the velocity profile at $r \leqslant 10^{\prime \prime}$ along the major-axis, and outside the central cuspy region along the minor-axis. These measurements are consistent with the Zwicky Catalog value of $-200 \pm 6 \mathrm{~km} \mathrm{~s}^{-1}$ (Falco et al. 1999).

\section{DYNAMICAL MODELS}

\subsection{Modeling Approach}

We fit our data with axisymmetric dynamical equilibrium models. The models are constructed using Schwarzschild's orbit superposition technique. Over the past decade, such models have become a standard in the field, and they have been applied and tested repeatedly with different software implementations on data for the galaxy M32 (e.g., van der Marel et al. 1998a; Cretton et al. 1999; Verolme et al. 2002; Valluri et al. 2004; Cappellari et al. 2006). van den Bosch \& de Zeeuw (2010) recently extended the modeling approach to include triaxial configurations. However, they found that M32 is actually best fit by models that are close to axisymmetric and edge-on. We therefore restrict our analysis to such models here.

Data sets of high spatial resolution obtained with the Hubble Space Telescope (HST; van der Marel et al. 1998b; Joseph et al. 2001) have demonstrated that M32 hosts a massive black hole in its center. Its mass is $M_{\mathrm{BH}}=(2.4 \pm 1.0) \times 10^{6} M_{\odot}$ (van den Bosch \& de Zeeuw 2010, consistent with determinations by previous authors). The observational setup for our new Keck data was optimized for spatial extent, and not spatial resolution. Rather than treat $M_{\mathrm{BH}}$ as a free parameter in our analysis, we therefore keep it fixed at $(2.4 \pm 1.0) \times 10^{6} M_{\odot}$ in all of our models.

We use the same modeling software as in van der Marel et al. (1998a), in which a constant $M / L$ is assumed to calculate the mass density distribution from the luminosity density.

The luminosity density is chosen to fit the observed major axis surface brightness profile. For this, we used the same $V$-band brightness profile as in van der Marel et al. (1998a), but we transformed this to the $I$-band using the known $V-I$ color (Lauer et al. 1998; Tonry et al. 2001). Slightly different from van der Marel et al. (1998a), we adopt a foreground extinction $A_{B}=0.35$ and projected axial ratio $q=0.76$ from van den Bosch \& de Zeeuw (2010). This makes our $M / L$ values directly comparable to theirs, since those authors also adopt the same distance as we do here.

Given this approach, the $M / L$ is the only free parameter to optimize the fit to the data. We construct models with different $M / L$. The best-fit model is identified as the one that yields residuals with the overall minimum $\chi^{2}$. We restrict our calculations here to models with a constant $M / L$ for several reasons: (1) such models are easiest to calculate; (2) such models have been found to adequately fit existing data sets for M32; (3) such models are a useful reference before considering more detailed modeling; and (4) such models can be used in many cases to prove the presence of a dark halo (namely, if no constant $M / L$ model can fit the data). 
Table 5

M32 Integrated Light Profiles for Velocity, Velocity Dispersion, and Gauss-Hermite Moments

\begin{tabular}{|c|c|c|c|c|c|c|c|c|c|c|c|c|c|c|c|}
\hline$r^{\mathrm{a}}$ & $d r^{\mathrm{b}}$ & $v^{\mathrm{c}}$ & $\Delta v^{\mathrm{d}}$ & $\sigma^{\mathrm{e}}$ & $\Delta \sigma^{\mathrm{f}}$ & $h_{3}{ }^{\mathrm{g}}$ & $\Delta h_{3}{ }^{\mathrm{h}}$ & $h_{4}{ }^{\mathrm{g}}$ & $\Delta h_{4}{ }^{\mathrm{h}}$ & $h_{5} \mathrm{~g}$ & $\Delta h_{5}{ }^{\mathrm{h}}$ & $h_{6}{ }^{\mathrm{g}}$ & $\Delta h_{6}{ }^{\mathrm{h}}$ & $\mathrm{S} / \mathrm{N}^{\mathrm{i}}$ & Mask Name \\
\hline \multicolumn{16}{|c|}{ M32 Major Axis $\left(\phi=160^{\circ}\right)$, Integrated Light } \\
\hline$-85^{\prime \prime} .0$ & $10^{\prime \prime} 0$ & -23.6 & 7.3 & 40.0 & 7.9 & - & - & - & - & - & - & - & - & 28 & - \\
\hline$-70^{\prime} .0$ & 5.0 & -19.5 & 6.0 & 40.6 & 6.9 & - & - & - & - & - & - & - & - & 33 & - \\
\hline-60.0 & 5.0 & -17.6 & 5.0 & 47.9 & 5.1 & 0.033 & 0.085 & 0.102 & 0.102 & -0.002 & 0.092 & -0.103 & 0.101 & 47 & - \\
\hline$-50^{\prime \prime} .0$ & $5 . .0$ & -19.2 & 2.5 & 41.1 & 2.8 & 0.047 & 0.058 & -0.067 & 0.073 & -0.028 & 0.062 & 0.081 & 0.071 & 80 & - \\
\hline$-40^{\prime} .0$ & 5.0 & -18.9 & 2.4 & 42.6 & 2.6 & -0.001 & 0.052 & -0.040 & 0.066 & 0.053 & 0.057 & 0.038 & 0.064 & 81 & - \\
\hline$-32^{\prime \prime} .5$ & 2.5 & -18.1 & 2.1 & 48.7 & 2.4 & 0.053 & 0.037 & -0.019 & 0.045 & -0.019 & 0.041 & 0.029 & 0.045 & 97 & - \\
\hline$-27 . .5$ & $2 . .5$ & -23.6 & 1.3 & 47.7 & 1.4 & 0.055 & 0.024 & 0.001 & 0.029 & -0.005 & 0.026 & 0.017 & 0.029 & 151 & - \\
\hline$-22^{\prime \prime} .5$ & $2 ! .5$ & -24.2 & 1.4 & 52.5 & 1.5 & 0.054 & 0.021 & 0.011 & 0.026 & -0.002 & 0.024 & -0.002 & 0.025 & 149 & - \\
\hline$-17^{\prime \prime} .5$ & 2.5 & -30.1 & 0.9 & 51.8 & 1.0 & 0.048 & 0.014 & 0.008 & 0.017 & 0.002 & 0.016 & 0.010 & 0.017 & 222 & - \\
\hline$-12^{\prime \prime} .5$ & $2 . .5$ & -36.2 & 0.8 & 54.4 & 0.9 & 0.048 & 0.012 & 0.017 & 0.014 & 0.022 & 0.013 & 0.001 & 0.014 & 250 & - \\
\hline$-7^{\prime \prime} .5$ & $2 ! .5$ & -43.8 & 0.6 & 55.7 & 0.6 & 0.050 & 0.008 & 0.016 & 0.009 & 0.019 & 0.009 & 0.002 & 0.009 & 360 & - \\
\hline$-4^{\prime \prime} .5$ & $0 ! .5$ & -44.9 & 0.9 & 58.8 & 1.0 & 0.060 & 0.011 & 0.030 & 0.013 & 0.000 & 0.012 & -0.015 & 0.013 & 240 & - \\
\hline$-3 . \prime 5$ & 0.5 & -44.9 & 0.7 & 59.7 & 0.8 & 0.059 & 0.009 & 0.014 & 0.011 & 0.003 & 0.010 & 0.001 & 0.011 & 287 & - \\
\hline-2.5 & $0 . .5$ & -45.7 & 0.8 & 60.8 & 0.9 & 0.058 & 0.009 & 0.024 & 0.011 & 0.006 & 0.011 & -0.009 & 0.012 & 258 & - \\
\hline$-1^{\prime \prime} .9$ & $0 . ' 6$ & -43.4 & 1.1 & 66.2 & 1.2 & 0.066 & 0.011 & 0.039 & 0.014 & -0.009 & 0.013 & -0.026 & 0.014 & 199 & Major_6 \\
\hline$-1^{\prime \prime} .9$ & $0 . ' 6$ & -44.9 & 1.1 & 62.8 & 1.2 & 0.067 & 0.012 & 0.034 & 0.014 & -0.009 & 0.014 & -0.019 & 0.014 & 199 & Major_5 \\
\hline$-1^{\prime \prime} 3$ & $0 . ' 6$ & -45.0 & 0.9 & 62.0 & 1.0 & 0.064 & 0.010 & 0.030 & 0.013 & -0.006 & 0.012 & -0.015 & 0.013 & 228 & Major_3 \\
\hline$-1^{\prime \prime} .3$ & $0 . ' 6$ & -45.3 & 0.9 & 62.2 & 1.0 & 0.067 & 0.010 & 0.028 & 0.012 & -0.004 & 0.012 & -0.010 & 0.012 & 236 & Major_4 \\
\hline$-0^{\prime \prime} 9$ & $0 . ' 6$ & -42.8 & 1.0 & 70.1 & 1.0 & 0.069 & 0.009 & 0.014 & 0.011 & 0.002 & 0.011 & -0.003 & 0.011 & 236 & Major_2 \\
\hline-0.7 & $0 ! .6$ & -22.6 & 0.9 & 81.3 & 0.9 & 0.056 & 0.006 & 0.010 & 0.007 & -0.027 & 0.007 & -0.009 & 0.007 & 312 & Major_6 \\
\hline-0.7 & $0 . ' 6$ & -23.7 & 0.8 & 79.1 & 0.8 & 0.062 & 0.006 & 0.015 & 0.007 & -0.024 & 0.007 & -0.011 & 0.007 & 319 & Major_5 \\
\hline$-0^{\prime \prime} 1$ & $0 . ' 6$ & -7.5 & 0.7 & 87.9 & 0.7 & 0.040 & 0.005 & -0.017 & 0.005 & -0.016 & 0.005 & 0.014 & 0.005 & 399 & Major_3 \\
\hline$-0^{\prime \prime} 1$ & $0 ! .6$ & -5.1 & 0.7 & 89.4 & 0.7 & 0.034 & 0.004 & -0.016 & 0.005 & -0.018 & 0.005 & 0.014 & 0.005 & 415 & Major_4 \\
\hline $0 ! 3$ & $0 ! .6$ & 14.7 & 0.9 & 87.3 & 0.9 & -0.054 & 0.006 & -0.012 & 0.006 & 0.029 & 0.007 & 0.009 & 0.007 & 322 & Major_2 \\
\hline 0.5 & 0.5 & 22.9 & 0.8 & 81.6 & 0.9 & -0.047 & 0.006 & 0.003 & 0.007 & 0.022 & 0.007 & -0.004 & 0.007 & 320 & Major_6 \\
\hline 0.5 & 0.5 & 23.8 & 0.8 & 79.3 & 0.8 & -0.056 & 0.006 & -0.001 & 0.007 & 0.027 & 0.007 & 0.002 & 0.007 & 326 & Major_5 \\
\hline $1^{\prime \prime} 0$ & 0.6 & 44.3 & 0.8 & 66.2 & 0.8 & -0.097 & 0.008 & 0.022 & 0.010 & 0.048 & 0.009 & -0.012 & 0.010 & 280 & Major_3 \\
\hline $1^{\prime \prime} 1$ & $0 . ' 6$ & 46.2 & 0.8 & 66.2 & 0.8 & -0.087 & 0.008 & 0.007 & 0.010 & 0.038 & 0.009 & 0.005 & 0.010 & 278 & Major_4 \\
\hline $1^{\prime \prime} 0$ & $0 . ' 6$ & 46.6 & 1.2 & 61.9 & 1.3 & -0.097 & 0.014 & 0.014 & 0.017 & 0.049 & 0.016 & -0.001 & 0.017 & 176 & Major_2 \\
\hline $1^{\prime \prime} 7$ & $0 . ' 6$ & 43.5 & 1.1 & 66.5 & 1.1 & -0.081 & 0.011 & 0.016 & 0.013 & 0.035 & 0.013 & -0.012 & 0.013 & 204 & Major_6 \\
\hline $1^{\prime \prime} .7$ & $0 . ' 6$ & 44.3 & 1.1 & 64.6 & 1.1 & -0.103 & 0.011 & 0.026 & 0.014 & 0.056 & 0.013 & -0.020 & 0.014 & 204 & Major_5 \\
\hline $2^{\prime \prime} .5$ & $0 ! .5$ & 46.3 & 0.6 & 61.3 & 0.7 & -0.091 & 0.007 & 0.021 & 0.009 & 0.038 & 0.008 & -0.010 & 0.009 & 337 & - \\
\hline $3^{\prime \prime} .5$ & $0 ! .5$ & 45.3 & 1.0 & 59.6 & 1.1 & -0.092 & 0.012 & 0.005 & 0.015 & 0.041 & 0.014 & 0.005 & 0.015 & 207 & - \\
\hline $4 . .5$ & $0 ! 5$ & 44.7 & 0.9 & 58.2 & 1.0 & -0.087 & 0.011 & 0.010 & 0.014 & 0.030 & 0.013 & 0.004 & 0.014 & 229 & - \\
\hline $7^{\prime \prime} .5$ & $2 ! .5$ & 42.7 & 0.5 & 56.2 & 0.6 & -0.089 & 0.007 & 0.013 & 0.009 & 0.032 & 0.008 & 0.000 & 0.009 & 384 & - \\
\hline $12^{\prime \prime} .5$ & $2 ! .5$ & 33.0 & 0.8 & 56.5 & 0.9 & -0.076 & 0.011 & 0.014 & 0.013 & 0.028 & 0.013 & -0.002 & 0.013 & 250 & - \\
\hline $17^{\prime \prime} .5$ & $2 ! .5$ & 28.1 & 1.0 & 54.1 & 1.1 & -0.090 & 0.015 & 0.016 & 0.018 & 0.053 & 0.016 & -0.009 & 0.018 & 205 & - \\
\hline $22^{\prime \prime} .5$ & $2 . .5$ & 23.4 & 1.3 & 51.6 & 1.4 & -0.077 & 0.021 & 0.015 & 0.025 & 0.049 & 0.023 & 0.000 & 0.025 & 155 & - \\
\hline $27 . .5$ & $2 . .5$ & 21.9 & 1.7 & 53.4 & 1.8 & -0.058 & 0.024 & 0.006 & 0.030 & 0.037 & 0.027 & -0.004 & 0.029 & 128 & - \\
\hline $32 . .5$ & $2 ! .5$ & 22.0 & 5.5 & 46.5 & 5.9 & -0.099 & 0.102 & -0.013 & 0.125 & 0.032 & 0.112 & 0.019 & 0.123 & 36 & - \\
\hline $40^{\prime \prime} 0$ & $5 . .0$ & 16.2 & 2.2 & 47.6 & 2.3 & -0.068 & 0.039 & -0.026 & 0.048 & 0.039 & 0.043 & 0.028 & 0.047 & 95 & - \\
\hline $500^{\prime \prime} 0$ & 5.0 & 16.9 & 3.4 & 43.6 & 3.7 & -0.105 & 0.070 & -0.020 & 0.086 & 0.108 & 0.075 & 0.034 & 0.084 & 63 & - \\
\hline $60^{\prime \prime} 0$ & 5.0 & 15.8 & 4.4 & 45.6 & 4.9 & -0.046 & 0.083 & -0.074 & 0.101 & 0.006 & 0.089 & 0.105 & 0.099 & 52 & - \\
\hline $700^{\prime} 0$ & $5 . .0$ & 14.7 & 5.9 & 42.4 & 6.4 & - & - & - & - & - & - & - & - & 39 & - \\
\hline $80 .{ }^{\prime \prime} 0$ & 5.0 & 18.7 & 7.5 & 29.0 & 9.8 & - & - & - & - & - & - & - & - & 25 & - \\
\hline \multicolumn{16}{|c|}{ M32 Minor Axis $\left(\phi=250^{\circ}\right)$, Integrated Light } \\
\hline$-40^{\prime} \cdot 0$ & 5.0 & -3.7 & 2.0 & 41.0 & 2.5 & -0.119 & 0.077 & -0.119 & 0.094 & 0.068 & 0.083 & 0.087 & 0.093 & 67 & - \\
\hline$-32 . .5$ & 2.5 & -2.1 & 2.0 & 39.3 & 2.3 & -0.189 & 0.074 & -0.189 & 0.092 & 0.168 & 0.080 & 0.418 & 0.091 & 63 & - \\
\hline$-27 . .5$ & $2 ! .5$ & -0.9 & 1.8 & 49.4 & 2.2 & -0.061 & 0.050 & -0.061 & 0.060 & 0.033 & 0.056 & 0.051 & 0.060 & 69 & - \\
\hline$-22^{\prime \prime} .5$ & $2 ! .5$ & 0.9 & 1.5 & 51.1 & 1.9 & -0.017 & 0.042 & -0.017 & 0.049 & 0.030 & 0.046 & 0.021 & 0.050 & 79 & - \\
\hline$-17^{\prime \prime} .5$ & $2 . .5$ & -0.2 & 1.0 & 52.8 & 1.3 & -0.034 & 0.027 & -0.034 & 0.032 & 0.027 & 0.030 & -0.008 & 0.032 & 118 & - \\
\hline$-12^{\prime \prime} .5$ & 2.5 & -0.8 & 0.9 & 55.9 & 1.2 & -0.035 & 0.023 & -0.035 & 0.027 & 0.013 & 0.025 & 0.051 & 0.027 & 129 & - \\
\hline$-7^{\prime \prime} .5$ & 2.5 & 0.5 & 0.6 & 60.2 & 0.8 & 0.005 & 0.013 & 0.005 & 0.016 & 0.003 & 0.015 & -0.008 & 0.016 & 193 & - \\
\hline$-4^{\prime \prime} .5$ & 0.5 & 3.0 & 0.9 & 61.9 & 1.2 & -0.003 & 0.019 & -0.003 & 0.023 & 0.000 & 0.022 & -0.033 & 0.023 & 128 & - \\
\hline$-3 . .5$ & 0.5 & 3.4 & 0.8 & 65.2 & 1.0 & -0.009 & 0.015 & -0.009 & 0.018 & 0.008 & 0.018 & -0.012 & 0.018 & 156 & - \\
\hline-2.5 & $0 ! .5$ & 4.6 & 0.6 & 69.0 & 0.8 & 0.000 & 0.011 & 0.000 & 0.012 & 0.006 & 0.012 & -0.022 & 0.013 & 211 & - \\
\hline$-1^{\prime \prime} .0$ & 0.6 & 9.3 & 0.6 & 79.9 & 0.7 & -0.006 & 0.008 & -0.006 & 0.010 & 0.002 & 0.010 & -0.005 & 0.010 & 241 & Minor_3 \\
\hline$-1^{\prime \prime} .0$ & $0 . ' 6$ & 10.4 & 0.6 & 78.9 & 0.7 & -0.021 & 0.008 & -0.021 & 0.009 & 0.004 & 0.010 & -0.003 & 0.010 & 247 & Minor_4 \\
\hline $0^{\prime \prime} 1$ & $0 ! .6$ & 11.0 & 0.5 & 87.4 & 0.6 & -0.035 & 0.006 & -0.035 & 0.007 & 0.013 & 0.007 & 0.016 & 0.007 & 323 & Minor_3 \\
\hline 0.2 & $0 . ' 6$ & 12.0 & 0.5 & 88.4 & 0.6 & -0.032 & 0.006 & -0.032 & 0.006 & 0.010 & 0.006 & 0.016 & 0.007 & 342 & Minor_4 \\
\hline $1^{\prime \prime} 3$ & $0 . ' 6$ & 8.1 & 0.6 & 77.4 & 0.8 & -0.020 & 0.009 & -0.020 & 0.011 & 0.009 & 0.011 & -0.006 & 0.011 & 222 & Minor_3 \\
\hline $1^{\prime \prime} 4$ & $0 . ' 6$ & 7.6 & 0.6 & 76.5 & 0.8 & -0.017 & 0.009 & -0.017 & 0.011 & 0.012 & 0.011 & -0.002 & 0.011 & 219 & Minor_4 \\
\hline $2^{\prime \prime} .5$ & 0.5 & 3.6 & 0.6 & 68.0 & 0.8 & -0.002 & 0.011 & -0.002 & 0.013 & 0.002 & 0.013 & -0.021 & 0.014 & 196 & - \\
\hline $3^{\prime \prime} .5$ & 0.5 & 1.7 & 0.8 & 64.8 & 1.0 & -0.012 & 0.016 & -0.012 & 0.018 & 0.018 & 0.018 & -0.028 & 0.018 & 151 & - \\
\hline
\end{tabular}


Table 5

(Continued)

\begin{tabular}{|c|c|c|c|c|c|c|c|c|c|c|c|c|c|c|c|}
\hline$r^{\mathrm{a}}$ & $d r^{\mathrm{b}}$ & $v^{\mathrm{c}}$ & $\Delta v^{\mathrm{d}}$ & $\sigma^{\mathrm{e}}$ & $\Delta \sigma^{\mathrm{f}}$ & $h_{3}{ }^{\mathrm{g}}$ & $\Delta h_{3}{ }^{\mathrm{h}}$ & $h_{4}{ }^{\mathrm{g}}$ & $\Delta h_{4}{ }^{\mathrm{h}}$ & $h_{5}{ }^{\mathrm{g}}$ & $\Delta h_{5}{ }^{\mathrm{h}}$ & $h_{6}{ }^{g}$ & $\Delta h_{6}{ }^{\mathrm{h}}$ & $\mathrm{S} / \mathrm{N}^{\mathrm{i}}$ & Mask Name ${ }^{j}$ \\
\hline 4.5 & 0.5 & 1.2 & 1.0 & 63.5 & 1.3 & 0.009 & 0.019 & 0.009 & 0.023 & -0.001 & 0.022 & -0.031 & 0.023 & 125 & - \\
\hline $7^{\prime \prime} .5$ & $2 . .5$ & 0.2 & 0.6 & 59.7 & 0.8 & 0.010 & 0.014 & 0.010 & 0.016 & 0.000 & 0.016 & -0.011 & 0.016 & 187 & - \\
\hline $12^{\prime \prime} .5$ & $2 . .5$ & -0.3 & 0.9 & 55.7 & 1.2 & -0.009 & 0.022 & -0.009 & 0.026 & -0.002 & 0.025 & -0.023 & 0.026 & 127 & - \\
\hline $17^{\prime \prime} .5$ & 2 ..5 & -1.6 & 1.0 & 51.4 & 1.3 & 0.000 & 0.028 & 0.000 & 0.033 & 0.004 & 0.031 & -0.002 & 0.033 & 113 & - \\
\hline $22^{\prime \prime} .5$ & $2 . .5$ & -5.0 & 1.4 & 48.8 & 1.9 & -0.012 & 0.044 & -0.012 & 0.053 & 0.006 & 0.049 & 0.011 & 0.053 & 79 & - \\
\hline $27 . .5$ & $2 . .5$ & -4.9 & 1.8 & 48.3 & 2.3 & -0.082 & 0.053 & -0.082 & 0.064 & 0.096 & 0.059 & 0.022 & 0.064 & 67 & - \\
\hline $32 . .5$ & 2 ..5 & -4.5 & 1.9 & 47.2 & 2.4 & -0.059 & 0.059 & -0.059 & 0.071 & 0.063 & 0.065 & 0.060 & 0.071 & 64 & - \\
\hline $40^{\prime \prime} 0$ & 5..0 & 2.6 & 2.9 & 42.3 & 3.9 & 0.022 & 0.117 & 0.022 & 0.142 & -0.020 & 0.126 & -0.002 & 0.141 & 37 & - \\
\hline $50 . .0$ & 5..0 & 2.1 & 2.7 & 41.9 & 3.6 & -0.040 & 0.107 & -0.040 & 0.131 & -0.004 & 0.114 & 0.062 & 0.128 & 42 & - \\
\hline $60 . .0$ & 5..0 & -2.5 & 4.0 & 49.6 & 5.3 & - & - & - & - & - & - & - & - & 30 & - \\
\hline $75^{\prime \prime} .0$ & $10^{\prime \prime} 0$ & -2.2 & 3.9 & 39.0 & 4.8 & - & - & - & - & - & - & - & - & 28 & - \\
\hline
\end{tabular}

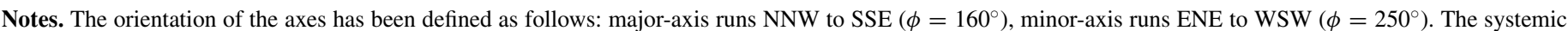
velocity of M32 is measured as $-199.7 \pm 0.5 \mathrm{~km} \mathrm{~s}^{-1}$ along the major-axis and $-200.1 \pm 1.8 \mathrm{~km} \mathrm{~s}^{-1}$ along the minor-axis.

${ }^{a}$ Projected distance along the axis in arcseconds.

b Spatial half-width of the bin in arcseconds.

${ }^{c}$ Measured LOS velocity in $\mathrm{km} \mathrm{s}^{-1}$.

${ }^{\mathrm{d}}$ Scaled Poisson error in measured LOS velocity in $\mathrm{km} \mathrm{s}^{-1}$ (where the scale factor $f=3.8$ and 2.1 for the major- and minor-axis, respectively).

e Measured velocity dispersion in $\mathrm{km} \mathrm{s}^{-1}$.

${ }^{\mathrm{f}}$ Scaled Poisson error in measured velocity dispersion in $\mathrm{km} \mathrm{s}^{-1}$ (where the scale factor $f=2.8$ and 1.9 for the major- and minor-axis, respectively).

g Gauss-Hermite moments for spectra with $\mathrm{S} / \mathrm{N} \gtrsim 40$.

h Scaled Poisson error in the Gauss-Hermite moments (where the scale factor $f=1.9$ and 2.1 for the major- and minor-axis, respectively.

${ }^{\mathrm{i}}$ Average $\mathrm{S} / \mathrm{N}$ per pixel of the spectrum.

j Mask names for the spectra that were not co-added (i.e., spectra located at $\left|r_{\mathrm{M} 32}\right| \leqslant 2^{\prime \prime}$ ).

\subsection{Data-model Comparison: Integrated Light}

The predictions of the best-fit model are compared to the long-slit integrated light measurements in Figures 12 and 13, for the major and minor axis, respectively.

The data-model comparison shows that integrated light kinematics for M32 are well-fit by an axisymmetric constant $M / L$ model (with a central black hole). While the fit is not perfect $\left(\chi^{2} / N_{\mathrm{DF}}=2.35\right)$, all of the trends in the data as a function of radius are reasonably well matched by the model.

The finding that a constant $M / L$ model adequately fits integrated-light kinematical data is consistent with what has been found by previous authors. However, our work extends this result to a radius that is three or more times larger than the region assessed by prior studies. This is a non-trivial finding, since one might have expected to start seeing the tell-tale signs of a possible dark halo at $\sim 3 r_{\text {eff }}$. But no such signs are evident.

The (I-band) mass-to-light ratio of our best-fit model is $M / L=1.24$. van den Bosch \& de Zeeuw (2010) found acceptable (triaxial) models in the range $M / L=1.4 \pm 0.2$. So while our best-fit value is lower than preferred by those authors, it is within the allowed range. The fact that, if anything, our fits over a much larger radial range yield a lower $M / L$ is important. If in reality the velocities of M32 stars were elevated at large radii because of the presence of a dark halo, then fitting a constant $M / L$ model should yield higher $M / L$ values when data at increasing radii are included.

\subsection{Data-model Comparison: Discrete Velocities}

The predictions of the best-fit model are compared to the $V$ and $\sigma$ inferred from the discrete velocity measurements (blue open dots) in Figures 14 and 15, for the major and minor axis, respectively. The long-slit data (black solid dots) are also included for comparison.

A three-integral model has the freedom to change its dynamical structure with radius. It is therefore worthwhile to consider for comparison simpler models that do not have this freedom. The green curves in Figure 14 show the large radii predictions of a two-integral model with a distribution function of the form $f\left(E, L_{z}\right)$. The Jeans equations of hydrostatic equilibrium can be explicitly solved for such models, making them a simple starting point for many analyses. They have been successfully applied to the case of M32 in many prior studies, and here we have used the same software as in van der Marel et al. (1994a) to calculate their predictions. The dotted green curves are for the same geometry and $M / L$ as in our best-fitting orbit superposition model. We adopted a parameter $k=0.57$ (defined in, e.g., van der Marel et al. 1994a) to split the azimuthal motion into ordered and random components.

On the major axis, in the area around the ends of our long slits $\left(40^{\prime \prime}-100^{\prime \prime}\right.$ from the center), the two-integral model predictions are very similar to those from our best-fitting three-integral model (solid red curves). On the minor axis, the three-integral model predictions for the dispersion are higher than for the twointegral model. This may be because (1) the best-fitting threeintegral model has a dynamical structure that differs from a two-integral model; or (2) the three-integral model predictions are obtained from Gaussian fits to model LOSVDs, whereas the two-integral model predictions are true dispersions. The long-dashed green curves in Figure 15 show the minor-axis predictions for a two-integral model with a $21 \%$ higher value of $M / L$. These appear more similar to the three-integral model predictions.

The purpose of the green two-integral curves in Figures 14 and 15 is to show how the kinematics fall with radius in a constant $M / L$ model in which the dynamical structure itself does not vary with radius. The gradient at large radii should not depend much on the dynamical structure itself, as long as it is independent of radius. The discrete velocity data points (blue points) at the large radii do not follow this nominal behavior. In particular, the velocity dispersions in the four outermost data points are higher than expected. The two-integral models 


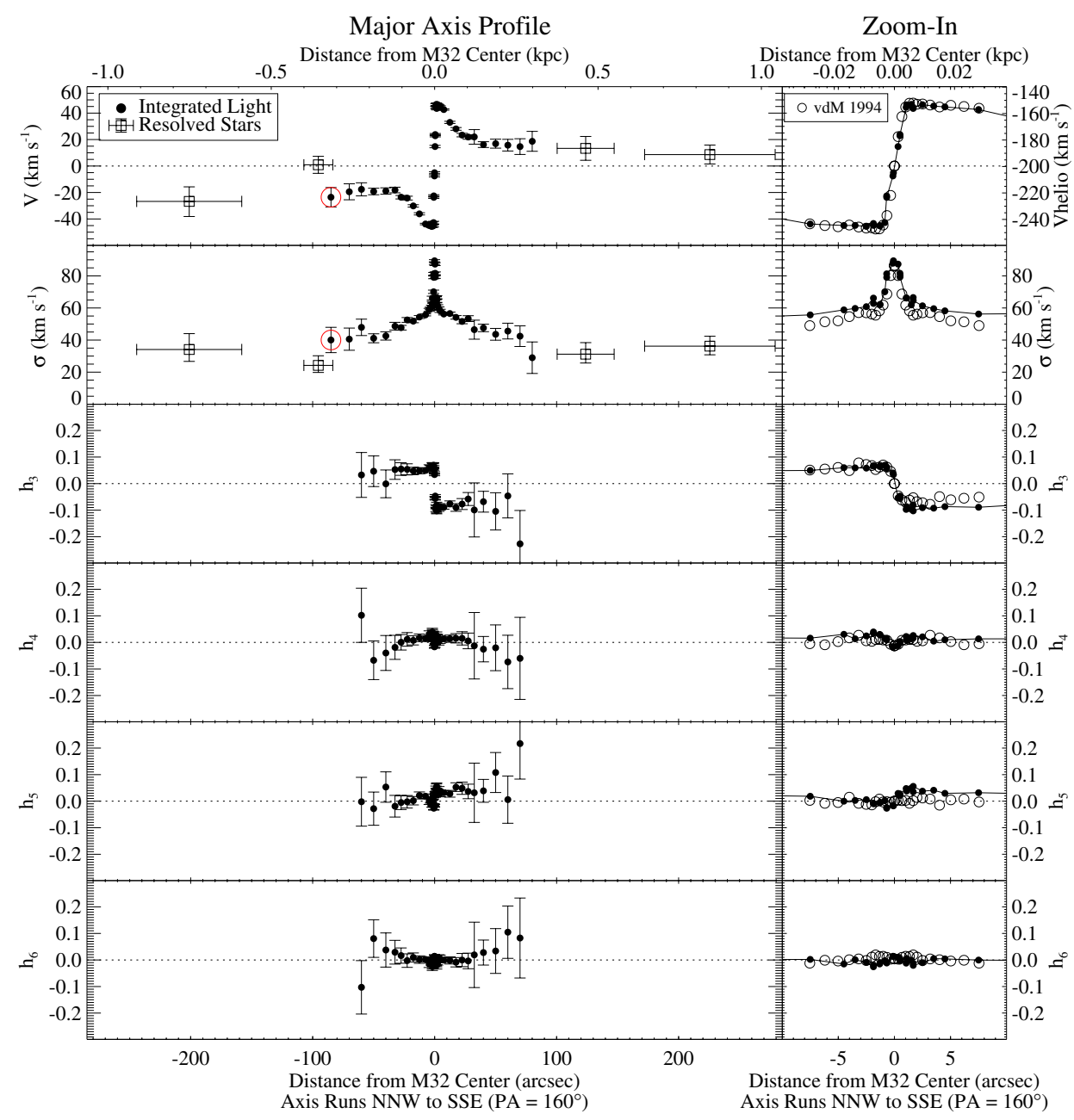

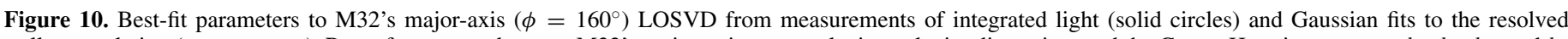

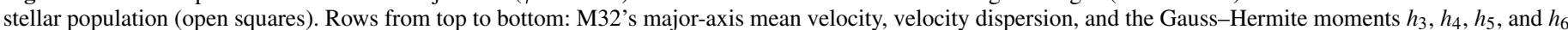

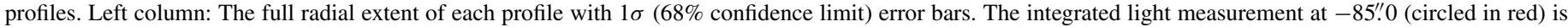

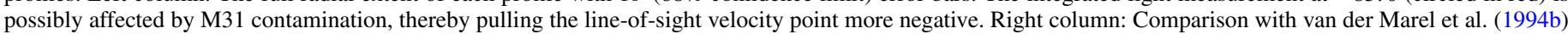

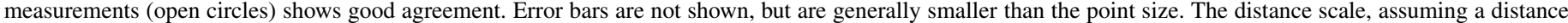
to M32 of $785 \mathrm{kpc}$, is $1^{\prime \prime}=4 \mathrm{pc}$.

(A color version of this figure is available in the online journal.)

(using the higher $M / L$ on the minor axis) predict, on average, $\sigma=24.7 \mathrm{~km} \mathrm{~s}^{-1}$ at these radii. By contrast, the observed weighted average dispersion is $33.0 \pm 3.5 \mathrm{~km} \mathrm{~s}^{-1}$, which is higher by a statistically significant $2.4 \sigma$. This suggests an increasing $M / L$ with radius (i.e., the presence of a dark halo), unless the dynamical structure of M32 changes with radius beyond the edges of our long slits.

The three-integral orbit superposition modeling approach automatically adjusts the dynamical structure as necessary to best fit all of the available data. The red dashed curves in Figures 14 and 15 show the predictions thus obtained. For each side of the galaxy (major or minor axis, positive or negative radius), there are two data points. The predictions for these data points are connected by a straight line. These predictions correspond to averages over broad wedges on the sky, and not small apertures as was the case for the integrated-light predictions. This affects primarily the rotation velocity, which is smaller when averaged over a wedge than on the major axis itself.

Given the significant scatter between the discrete velocity data points, the three-integral model fits the data reasonably well. In particular, the model predictions significantly exceed the nominal model fall-off indicated by the two-integral models.
For the four outermost data points, the predicted dispersion is $32.7 \mathrm{~km} \mathrm{~s}^{-1}$, consistent with the weighted average $33.0 \pm$ $3.5 \mathrm{~km} \mathrm{~s}^{-1}$ of the observed values. For the four inner data points, the predicted dispersion does not fit the average of the observed values, $38.6 \mathrm{~km} \mathrm{~s}^{-1}$ versus $24.9 \pm 2.3 \mathrm{~km} \mathrm{~s}^{-1}$, respectively. Apparently, a constant $M / L$ model cannot simultaneously reproduce the low dispersions observed in the inner wedges of Figure 5, while also reproducing the higher dispersions observed in the outer wedges.

The reason that the three-integral models predict higher dispersions than the two-integral models is due to a change in its dynamical structure. Inspection of the dynamical structure of the best-fitting three-integral model shows that it has increasing tangential anisotropy toward larger radii. This causes more motion to be observed along the line of sight direction. Moreover, models with tangential anisotropy tend to have flat-topped LOSVDs. For such LOSVDs, the dispersion of the best-fitting Gaussian (which is the observed quantity) exceeds the true dispersion (van der Marel \& Franx 1993). Both of these effects help the model to fit the observed dispersions at large radii.

To assess whether the tangential anisotropy of the best-fitting three-integral model is consistent with the data, it is necessary 


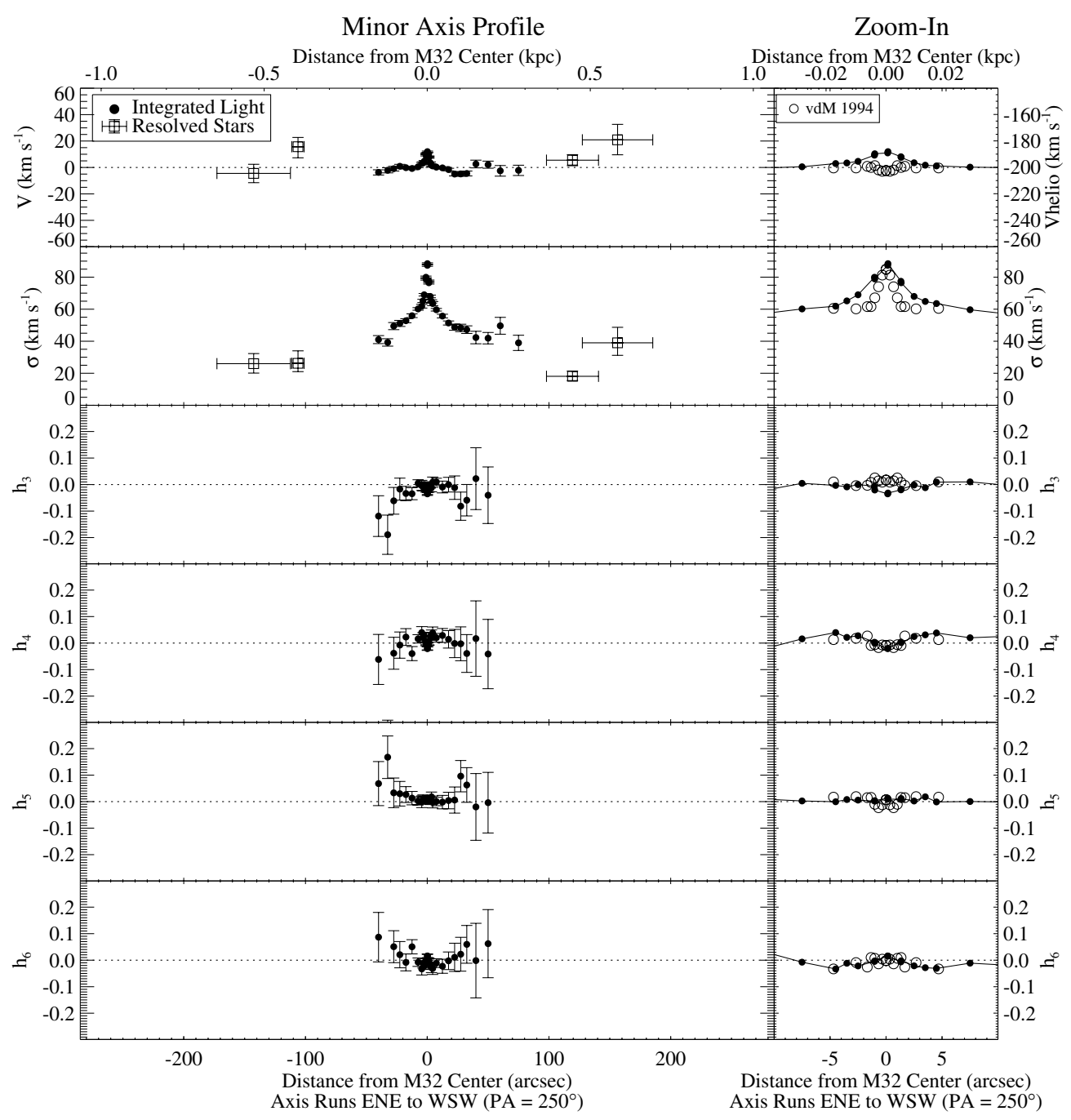

Figure 11. Same as Figure 10, but for M32's minor-axis $\left(\phi=250^{\circ}\right)$. The slight mismatch seen at the center in the mean velocity profile (an apparent peak in our data and an apparent dip in van der Marel et al. (1994b)), and the $h 3$ and $h 5$ profiles is an artifact that results from a slight mis-centering of the longslit in our observations and that of van der Marel et al. (1994b).

to measure the shape of the LOSVD at large radii (Carollo et al. 1995). On average, the outer wedges in Figure 5 each have only some 28 observed M32 stars in them, much too little to reliably determine the Gauss-Hermite moments of the LOSVD. Nonetheless, some important LOSVD shape information can be obtained from the data. Figure 16 shows the observed grand-total velocity histogram for the outer four wedges, with the M31 contribution (held fixed at the values in Figure 6) subtracted. The red curve is the prediction of the best-fitting three-integral model. The curves have similar widths, as was already clear from the preceding discussion. However, there are some subtle differences in the observed and predicted LOSVD shape.

The vertical dashed curves in Figure 16 show the escape velocity of the model at $R=200^{\prime \prime}$ in the equatorial plane $\left(72 \mathrm{~km} \mathrm{~s}^{-1}\right)$. The wings of the predicted LOSVD fall to zero around this velocity. To achieve a significant dispersion, the model creates a relatively flat-topped LOSVD within the regime bounded by the escape velocity. By contrast, the observed LOSVD histogram has a narrower core, and broader wings. In particular, there are $\sim 6$ stars on the positive velocity side of the LOSVD that move faster than the model escape velocity. If these stars are bound to M32, then M32 must have a dark halo.
However, alternative interpretations do exist. For one, it is possible that the excess stars at $v \approx 95 \mathrm{~km} \mathrm{~s}^{-1}$ in the M32 frame (i.e., $v_{\text {hel }} \approx-105 \mathrm{~km} \mathrm{~s}^{-1}$ ) are simply due to a co-moving group of M31 stars. Our simple model for the LOSVD of the M31 halo is a smooth Gaussian. Although noise in the histogram of M31 stars is not accounted for, we expect the noise to be higher at negative $v$ rather than positive $v$ because most M31 stars are at velocities much lower than M32 (see Figure 7). The fact that all bins for $v>100 \mathrm{~km} \mathrm{~s}^{-1}$ in Figure 16 are very close to zero, with little scatter, indicates there is very little shot noise from M31 stars there. Therefore, if the stars in the bin $80-100 \mathrm{~km} \mathrm{~s}^{-1}$ are due to M31 and not M32, then it is more likely the result of substructure in M31 than random noise in the M31 background. A second possibility is that we have reached a regime in M32 where tidal perturbations are playing a role. In this case, it would not be appropriate to interpret the excess stars in the context of an equilibrium model. The fact that the observed histogram in Figure 16 is not symmetric (there are excess stars only on the positive velocity side) seems more consistent with either of these interpretations than with evidence for a dark halo.

Other than the high-velocity tail of stars in Figure 16, our data-model comparison provides very little evidence that the 
Major-axis data-model comparison
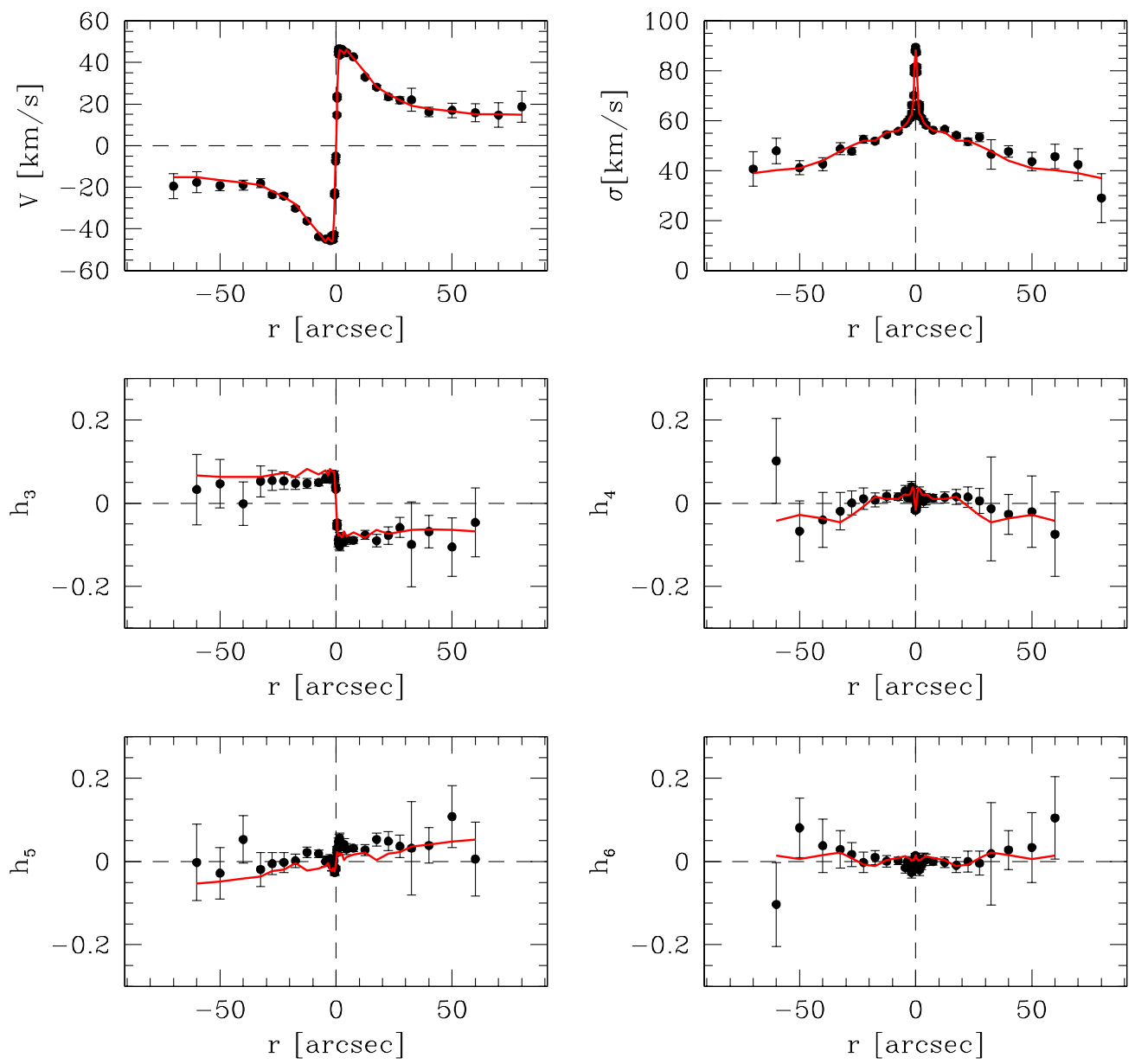

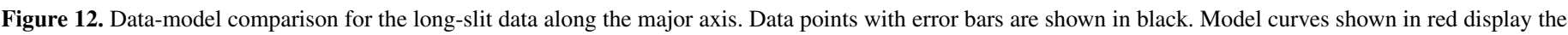

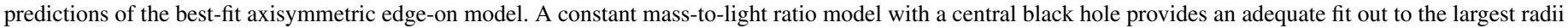
accessible with integrated light.

(A color version of this figure is available in the online journal.)

kinematics of M32 might be affected by tidal perturbations or non-equilibrium dynamics. The integrated-light measurements inside $100^{\prime \prime}$ have a smooth behavior, and closely follow the predictions of our equilibrium dynamical models. The discrete measurements at larger radii show significant scatter, but this is likely due to measurement errors, and not tidally induced. The velocity dispersion increases from the inner to the outer wedges on Figure 5, but this increase is similar on all four sides of the galaxy, and therefore is not easily attributed to tidal perturbations.

In summary, we find that the best-fitting three-integral constant $-M / L$ model with increasing tangential anisotropy toward large radii provides a sufficiently decent fit to the data in Figures 14, 15, and 16, such that we cannot rule out a model without a dark halo. However, by obtaining kinematics out to $\sim 8 r_{\text {eff }}$, we appear to have reached for the first time in M32 a regime where the observed velocity dispersion flattens out as a function of radius. In almost all of the other galaxies where this is seen, this is attributed to the influence of a dark halo, and in many cases this can be proven to be the only possible explanation.

We have not explicitly explored dynamical models that include a dark halo component. Given the added freedom of an $M / L$ that varies with radius, such models should certainly be able to fit the radial trends in the data (symmetrized over both sides of the galaxy). However, given the limited number of data points at the largest radii, it is unlikely that such models would be able to place strong constraints on the properties of the dark halo.

\section{SUMMARY AND DISCUSSION}

We have presented mean velocity, velocity dispersion, and higher order Gauss-Hermite moment profiles along M32's major and minor axes based on Keck/DEIMOS spectroscopic observations of the integrated light and the resolved stellar population. This study is the first to continuously transition between integrated light and the resolved stellar population in M32, or indeed in any galaxy. The kinematical profiles provide the most radially extensive spectroscopic coverage for any $\mathrm{cE}$ galaxy, with measurements of the resolved stellar population extending to a projected distance of $\sim 8 r_{I}^{\mathrm{eff}}$.

We have constructed axisymmetric three-integral dynamical models for M32 to interpret the new data. The integratedlight data out to $\sim 100^{\prime \prime}$ show falling dispersions with radius, which are well-fit by a constant $M / L$ model. The discrete velocity data between $100^{\prime \prime}$ and $200^{\prime \prime}$ reveal a regime where the observed velocity dispersion flattens out as a function of 
Minor-axis data-model comparison
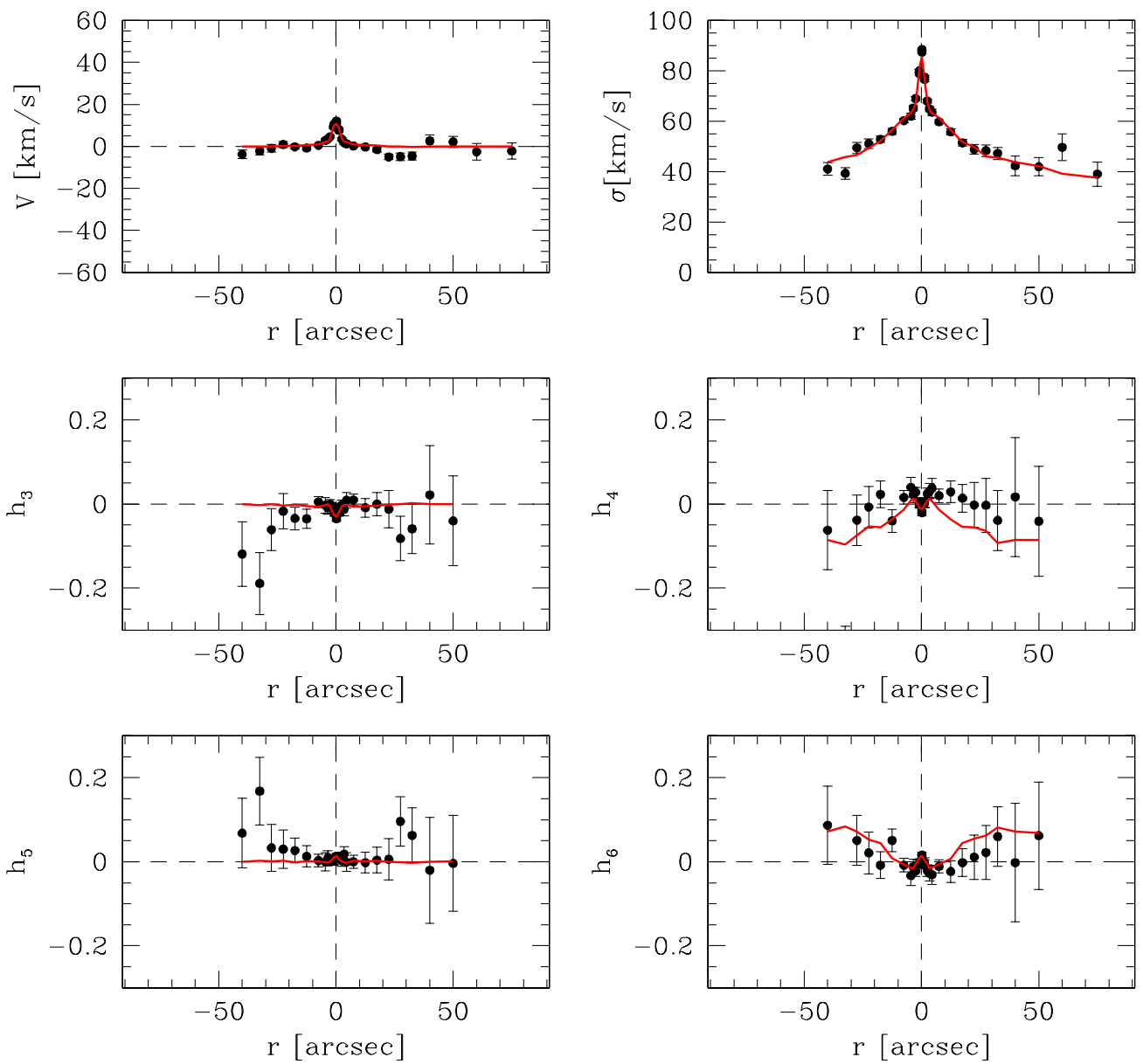

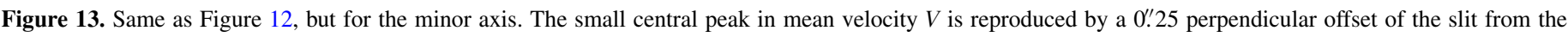
galaxy center.

(A color version of this figure is available in the online journal.)

radius. This can be plausibly interpreted as evidence for a dark halo. However, a constant $M / L$ model can fit all of the available data out to the largest radius probed, provided that there is increasing tangential anisotropy with radius in M32. The number of observed M32 stars at large radii is too small to directly constrain the anisotropy. A small number of fast moving stars at large radii suggests that a dark halo may better explain the data than tangential anisotropy, but these stars may also be due to substructure in the M31 halo, or tidal perturbations in the M32 outskirts.

As discussed in Section 1, it has been suggested that tides may have played a role in the formation of M32. One might expect tides to induce structural asymmetries at large radii, or other distortions from what one would normally expect in an equilibrium model. Indeed, it has long been known that M32's isophotes undergo a sharp twist and increase in ellipticity coincident with a break in the surface brightness profile at $\sim 5 r_{I}^{\text {eff }}$, plausibly as a result of tidal interaction with M31 (e.g., Kent 1987; Choi et al. 2002; Johnston et al. 2002). Our kinematical study, however, shows no strong corresponding distortions or asymmetries across this region. The lack of a strong gradient in the velocity and velocity dispersion profiles across the radius at which isophotal distortion occurs does not necessarily rule out the tidal distortion hypothesis. If M32 is interacting, then one plausible explanation for the absence of a strong observable signature in the line-of-sight kinematics is that the velocity distortion happens to be primarily in the plane of the sky. An alternative explanation to the tidal distortion hypothesis is that the observed isophotal distortions in M32 are intrinsic to its structure. Fasano \& Bonoli (1989) find an increase in ellipticity and isophotal twisting in the outer regions of about half of all of the isolated elliptical galaxies, and conclude that tides are unlikely to be the cause of the distortion seen in these galaxies. In order to determine if M32's kinematics are consistent with a tidal interaction hypothesis, a rigorous calculation of the impact of tides on the kinematical profile is needed.

K.M.H. was supported in part by the Lawrence Scholars Program at Lawrence Livermore National Laboratory (LLNL-JRNL-496754). E.K. and K.G. were supported through Hubble Fellowship grants 51256.01 and 51273.01, respectively, from the Space Telescope Science Institute, which is operated by the Association of Universities for Research in Astronomy, Inc., for NASA, under contract NAS 5-26555. P.G., K.M.H., and B.Y. acknowledge support from NSF grants AST0607852 and AST-1010039. J.S.K.'s research is supported in part by a grant from the STScI Director's Discretionary Research Fund. K.M.H. thanks STScI and Yale University for 

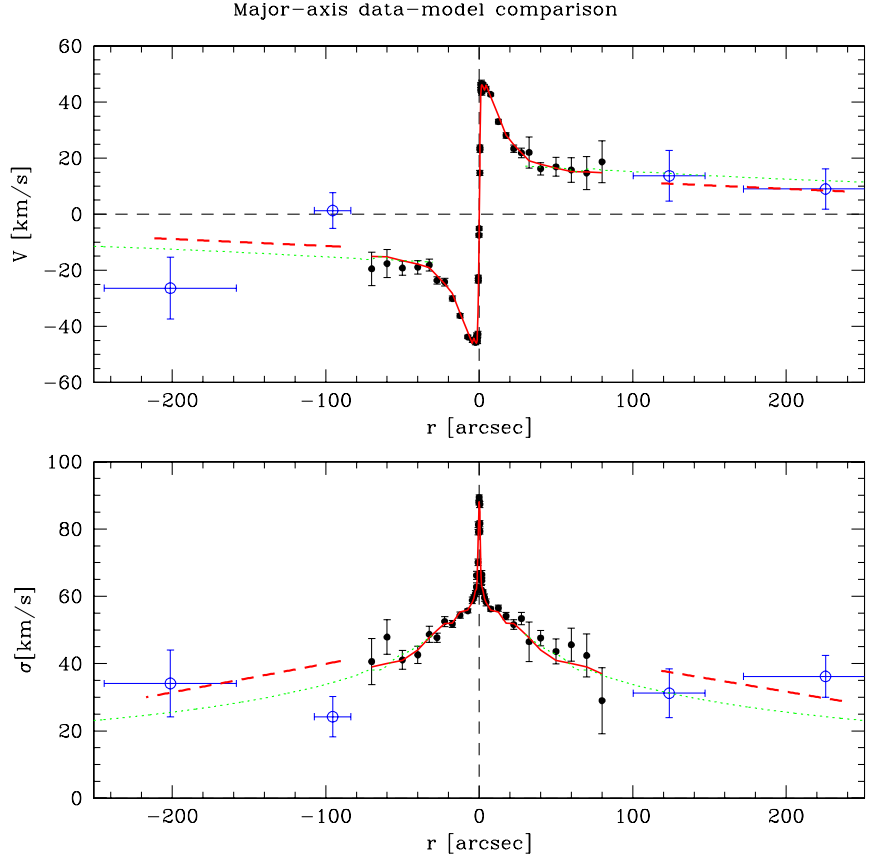

Figure 14. Data-model comparison for the Gaussian parameters $V$ and $\sigma$, along the major axis. Blue open data points are from fits to the discrete velocity measurement histograms. Solid black data points are from integratedlight measurements. Model curves shown in red display the predictions of the best-fit three-integral axisymmetric edge-on model. Model predictions for the integrated light data (corresponding to thin apertures) are connected by a solid red line. Model predictions for the discrete velocity data (corresponding to broad wedges, two on each side of the galaxy) are connected by a dashed red line. For comparison, dotted green curves at large radii show the major-axis predictions (not integrated over wedges) of a two-integral model with the same geometry and mass-to-light ratio.

(A color version of this figure is available in the online journal.)
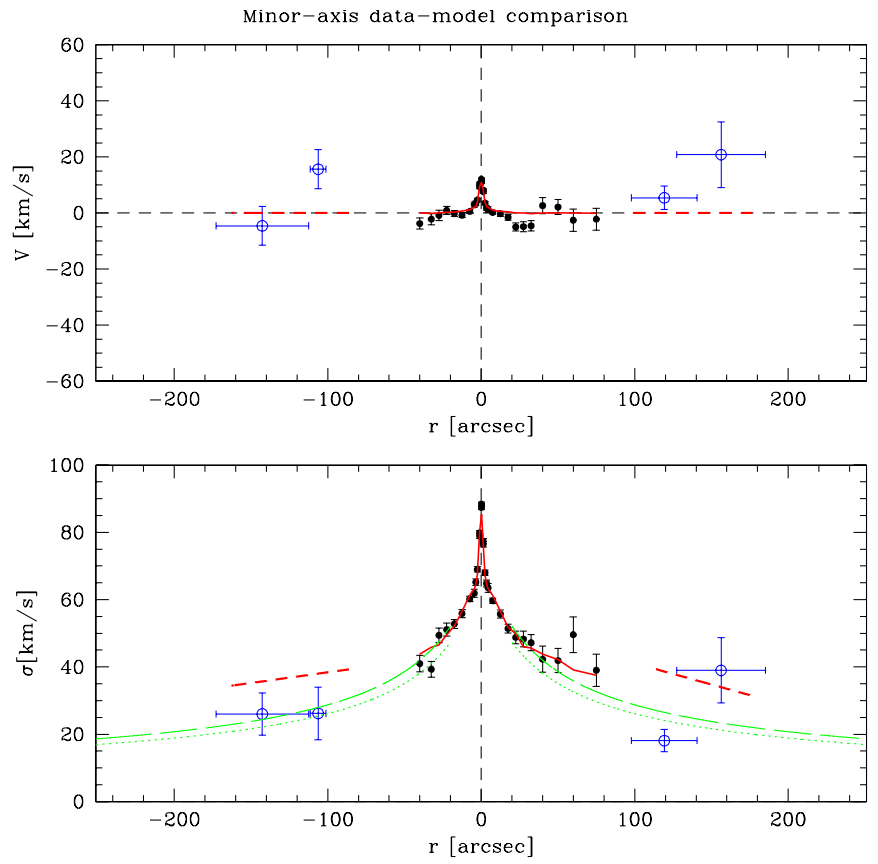

Figure 15. Same as Figure 14, but for the minor axis. The dotted green curves at large radii show the predictions of a two-integral model with the same geometry and mass-to-light ratio as the best-fit three-integral model (red). The long-dashed green curves show the predictions of a two-integral model with a $21 \%$ larger mass-to-light ratio.

(A color version of this figure is available in the online journal.)

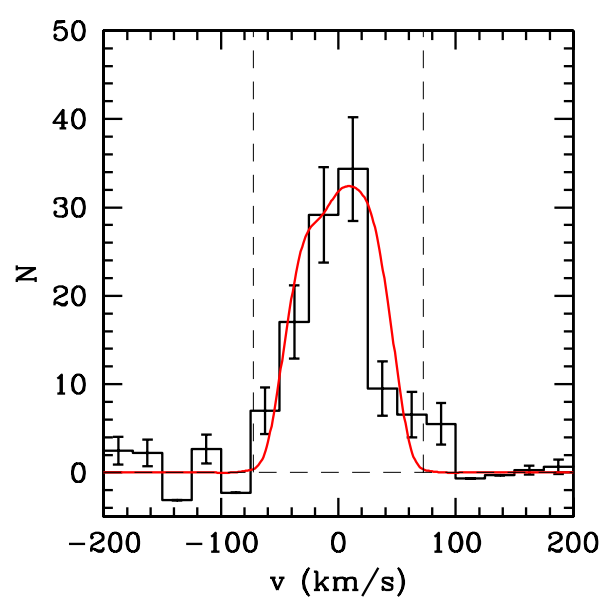

Figure 16. Observed grand-total velocity histogram for the outer four wedges in Figure 5, with the M31 contribution (held fixed at the values in Figure 6) subtracted. The red curve is the prediction of the best-fitting three-integral model. The horizontal axis shows the velocity in the M32 frame $\left(v_{\text {hel }}+\right.$ $200 \mathrm{~km} \mathrm{~s}^{-1}$ ). Vertical dashed curves at $\pm 72 \mathrm{~km} \mathrm{~s}^{-1}$ indicate the escape velocity of the model at $R=200^{\prime \prime}$ in the equatorial plane. The slight mismatch between the observed and predicted histograms may be interpreted either as evidence for an M32 dark halo, evidence for tidal perturbations in the M32 outskirts, or evidence for velocity substructure in the M31 halo.

(A color version of this figure is available in the online journal.)

their hospitality during her visits to carry out some of this work.

The authors wish to recognize and acknowledge the very significant cultural role and reverence that the summit of Mauna Kea has always had within the indigenous Hawaiian community. We are most fortunate to have the opportunity to conduct observations from this mountain.

Facility: Keck:II (DEIMOS)

\section{REFERENCES}

Bekki, K., Couch, W. J., Drinkwater, M. J., \& Gregg, M. D. 2001, ApJL, 557, L42

Bender, R., Kormendy, J., \& Dehnen, W. 1996, ApJL, 464, L123

Bertin, E., Mellier, Y., Radovich, M., et al. 2002, in ASP Conf. Ser. 281, Astronomical Data Analysis Software and Systems XI, ed. D. A. Bohlender, D. Durand, \& T. H. Handley (San Francisco, CA: ASP), 228

Binggeli, B., Sandage, A., \& Tammann, G. A. 1988, ARA\&A, 26, 509

Burkert, A. 1994, MNRAS, 266, 877

Cappellari, M., Bacon, R., Bureau, M., et al. 2006, MNRAS, 366, 1126

Cappellari, M., Emsellem, E., Bacon, R., et al. 2007, MNRAS, 379, 418

Carollo, C. M., de Zeeuw, P. T., van der Marel, R. P., Danziger, I. J., \& Qian, E. E. 1995, ApJL, 441, L25

Carter, D., \& Jenkins, C. R. 1993, MNRAS, 263, 1049

Chilingarian, I., Cayatte, V., Revaz, Y., et al. 2009, Sci, 326, 1379

Chilingarian, I. V., \& Bergond, G. 2010, MNRAS, 405, L11

Choi, P. I., Guhathakurta, P., \& Johnston, K. V. 2002, AJ, 124, 310

Coelho, P., Mendes de Oliveira, C., \& Cid Fernandes, R. 2009, MNRAS, 396,624

Cretton, N., de Zeeuw, P. T., van der Marel, R. P., \& Rix, H.-W. 1999, ApJS, 124,383

Davidge, T. J. 2000, PASP, 112, 1177

Davidge, T. J., Rigaut, F., Chun, M., et al. 2000, ApJL, 545, L89

Dorman, C. E., Guhathakurta, P., Fardal, M. A., et al. 2012, ApJ, 752, 147

Dressler, A., \& Richstone, D. O. 1988, ApJ, 324, 701D

Drinkwater, M. J., \& Gregg, M. D. 1998, MNRAS, 296, L15

Faber, S. M. 1973, AJ, 179, 423

Faber, S. M., Phillips, A. C., Kibrick, R. I., et al. 2003, Proc. SPIE, 4841, 1657

Falco, E. E., Kurtz, M. J., Geller, M. J., et al. 1999, PASP, 111, 438

Fasano, G., \& Bonoli, C. 1989, A\&AS, 79, 291

Ford, H. C., Jacoby, G. H., \& Jenner, D. C. 1978, ApJ, 223, 94

Gilbert, K. M., Fardal, M., Kalirai, J. S., et al. 2007, ApJ, 668, 245 
Goodman, J., \& Lee, H. M. 1989, ApJ, 337, 84

Graham, A. W. 2002, ApJL, 568, L13

Guhathakurta, P., Ostheimer, J. C., Gilbert, K. M., et al. 2005, preprint (astro-ph/0502366)

Guhathakurta, P., Rich, R. M., Reitzel, D. B., et al. 2006, AJ, 131, 2497

Howley, K. M., Geha, M., Guhathakurta, P., et al. 2008, ApJ, 683, 722

Huxor, A., Phillipps, S., Price, J., \& Harniman, R. 2010, arXiv:1009.3185

Irwin, M. J., Ferguson, A. M. N., Ibata, R. A., Lewis, G. F., \& Tanvir, N. R. 2005, ApJL, 621, L105

Jensen, J. B., Tonry, J. L., Barris, B. J., et al. 2003, ApJ, 583, 712

Johnston, K. V., Choi, P. I., \& Guhathakurta, P. 2002, AJ, 124, 127

Joseph, C. L., Merritt, D., Olling, R., et al. 2001, ApJ, 550, 668

Karachentsev, I. D., Karachentseva, V. E., Huchtmeier, W. K., \& Makarov, D. I. 2004, AJ, 127, 2031

Kent, S. M. 1987, ApJ, 266, 562

King, I. R., \& Kiser, J. 1973, ApJ, 181, 27

Kormendy, J. 1985, ApJ, 295, 73

Lauer, T. R., Faber, S. M., Ajhar, E. A., Grillmair, C. J., \& Scowen, P. A. 1998, AJ, 116, 2263

McConnachie, A. W., Irwin, M. J., Ferguson, A. M. N., et al. 2005, MNRAS, 356, 979

Mieske, S., Infante, L., Hilker, M., et al. 2005, A\&A, 430, L25

Monachesi, A., Trager, S. C., Lauer, T. R., et al. 2011, ApJ, 727, 55

Nieto, J.-L. 1990, in Dynamics and Interactions of Galaxies, ed. R. Wielen (Berlin: Springer), 258

Nieto, J.-L., \& Prugniel, P. 1987, A\&A, 186, 30

O’Connell, R. W. 1980, ApJ, 236, 430

Pritchet, C. J., \& van den Bergh, S. 1994, AJ, 107, 1730

Rix, H.-W., \& White, S. D. M. 1992, MNRAS, 254, 389
Rood, H. J. 1965, AJ, 70, 689

Rose, J. A., Arimoto, N., Caldwell, N., et al. 2005, AJ, 129, 712

Simon, J. D., \& Geha, M. 2007, ApJ, 670, 313

Smith Castelli, A. V., Faifer, F. R., Bassino, L. P., et al. 2009, BAAA, 52, 229S

Sohn, S. T., Majewski, S. R., Muñoz, R. R., et al. 2007, ApJ, 663, 960

Stetson, P. B. 1994, PASP, 106, 250

Toloba, E., Boselli, A., Cenarro, A. J., et al. 2011, A\&A, 526, A114

Tonry, J. L. 1984, ApJL, 283, L27

Tonry, J. L. 1987, ApJ, 322, 632

Tonry, J. L., Dressler, A., Blakeslee, J. P., et al. 2001, ApJ, 546, 68

Tremaine, S., Gebhardt, K., Bender, R., et al. 2002, ApJ, 574, 740

Valluri, M., Merritt, D., \& Emsellem, E. 2004, ApJ, 602, 66

van den Bosch, R. C. E., \& de Zeeuw, P. T. 2010, MNRAS, 401, 1770

van der Marel, R. P. 1994, MNRAS, 270, 271

van der Marel, R. P., Cretton, N., de Zeeuw, P. T., \& Rix, H. W. 1998a, ApJ 493, 613

van der Marel, R. P., de Zeeuw, P. T., \& Rix, H.-W. 1998b, ApJ, 488, 119

van der Marel, R. P., de Zeeuw, P. T., Rix, H.-W., \& Quinlan, G. D. 1997, Natur, 385,610

van der Marel, R. P., Evans, N. W., Rix, H.-W., White, S. D. M., \& de Zeeuw, T. 1994a, MNRAS, 271, 99

van der Marel, R. P., \& Franx, M. 1993, ApJ, 407, 525

van der Marel, R. P., Rix, H. W., Carter, D., et al. 1994b, MNRAS, 268,521

Verolme, E. K., Cappellari, M., Copin, Y., et al. 2002, MNRAS, 335,517

Walterbos, R. A. M., \& Kennicutt, R. C., Jr. 1987, A\&AS, 69, 311

Wirth, A., \& Gallagher, J. S., III 1984, ApJ, 282, 85

Ziegler, B. L., \& Bender, R. 1998, A\&A, 330, 819 